Geometry $\&$ Topology

Volume 9 (2005) 247-297

Published: 26 January 2005

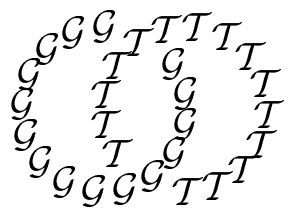

\title{
Knot and braid invariants from contact homology I
}

\author{
LENHARD NG \\ Department of Mathematics, Stanford University \\ Stanford, CA 94305, USA \\ Email: lng@math.stanford.edu \\ URL: http://alum.mit.edu/www/ng
}

\begin{abstract}
We introduce topological invariants of knots and braid conjugacy classes, in the form of differential graded algebras, and present an explicit combinatorial formulation for these invariants. The algebras conjecturally give the relative contact homology of certain Legendrian tori in five-dimensional contact manifolds. We present several computations and derive a relation between the knot invariant and the determinant.
\end{abstract}

\section{AMS Classification numbers Primary: 57M27}

Secondary: 53D35, 20F36

Keywords: Contact homology, knot invariant, braid representation, differential graded algebra

Proposed: Yasha Eliashberg

Seconded: Robion Kirby, Joan Birman
Received: 16 July 2004

Accepted: 13 January 2005 


\section{Introduction}

The study of invariants of Legendrian submanifolds in contact manifolds is currently a very active field of research. Holomorphic curve techniques stemming from the introduction of Symplectic Field Theory [7] have inspired a great deal of work on Legendrian isotopy invariants; papers in the subject include 3, 5, 6, 8, 9, 18, 23, among others. Most of these papers focus on Legendrian knots, and all deduce results in symplectic and contact geometry. Legendrian knots, however, form a restrictive class within the space of all knots, and Legendrian isotopy only makes sense in the presence of an ambient contact structure.

In this manuscript, we use ideas from Symplectic Field Theory to obtain new invariants of topological knots and braids. We note that holomorphic curves have been previously used to define knot invariants, through the work of Ozsváth and Szabó 21] on Heegaard Floer homology. Their approach, however, is very different from the one leading to the invariants in our paper, which is due to Eliashberg and can be briefly summarized as follows. A knot or braid yields in a natural way a Legendrian torus in a certain 5-dimensional contact manifold, and topological isotopy of the knot or braid results in Legendrian isotopy of the torus; thus, Legendrian invariants of the torus, and in particular relative contact homology, yield topological invariants for the original knot or braid.

We will discuss this correspondence further in Section 3, but the analytical underpinnings of the precise calculation of relative contact homology are still in progress. Instead, in the rest of the paper, we handle our new invariants algebraically, with no reference to contact geometry. The proofs of invariance, and all subsequent calculations, are completely combinatorial and algebraic.

We now describe our invariants. The starting point is a representation $\phi$ of the braid group $B_{n}$ as a group of algebra automorphisms of a tensor algebra with $n(n-1)$ generators. This representation, which extends ones considered previously by Magnus [16] and Humphries [12, is nearly faithful, and there is a slightly larger representation $\phi^{\text {ext }}$ which is faithful. The map $\phi$ then gives rise to a differential graded algebra (customarily abbreviated "DGA" in the subject) for any braid $B$, which we call the braid DGA, while $\phi^{\text {ext }}$ yields a larger algebra called the knot DGA.

There is an equivalence relation on DGAs first introduced by Chekanov [3] called stable tame isomorphism, which has been the subject of much work because of its importance in the theory of Legendrian invariants. The main results of this paper state that, up to stable tame isomorphism, the braid DGA of $B$ is an 
invariant of the conjugacy class of $B$, while the knot DGA of $B$ is an invariant of the knot which is the closure of $B$.

In particular, the graded homology of these DGAs, which is unchanged by stable tame isomorphism, gives an invariant of braid conjugacy classes and knots; we call this the contact homology of the braid or knot. Although the homology of a DGA is generally hard to compute, there is a relatively simple form for the 0-dimensional part $H C_{0}$ of the contact homology, and we can compute $H C_{0}$ explicitly for a large number of knots.

We concentrate on the invariant $H C_{0}$ because it is difficult in general to distinguish between stable tame isomorphism classes of DGAs, and because $H C_{0}$ seems to encode much of the information from the knot or braid DGA. It is sometimes also hard to tell when two knots or braids have isomorphic $H C_{0}$, but we can deduce numerical invariants from $H C_{0}$, called augmentation numbers, which are easy to calculate by computer.

Knot contact homology seems to be an invariant distinct from previously known knot invariants. On the one hand, it does not distinguish between mirrors, and a result in [19] states that, for two-bridge knots, $H C_{0}$ depends only on the determinant $\left|\Delta_{K}(-1)\right|$ of the knot; hence one might expect that knot contact homology is fairly weak as an invariant. On the other hand, there are pairs of knots which are distinguished by $H C_{0}$ (via augmentation numbers) but not by (any given one of) a litany of familiar knot invariants: Alexander polynomial, Jones polynomial, HOMFLY polynomial, Kauffman polynomial, signature, Khovanov invariant, and Ozsváth-Szabó invariant.

One classical invariant that is contained in knot contact homology is the determinant; in fact, the determinant is encoded by a linearization $H C_{*}^{\text {lin }}$ of $H C_{*}$. A further link between $H C_{0}(K)$ and the determinant is the existence of a surjection from $H C_{0}(K)$ to a ring $\mathbb{Z}[x] /(p(x))$, where $p$ is a certain polynomial determined by the homology of the double branched cover over $K$; this map is often an isomorphism.

Although knot contact homology is uniquely defined as a theory over $\mathbb{Z}_{2}$, it has different viable lifts to $\mathbb{Z}$, one of which is the theory described above. However, there is another, inequivalent, lift to $\mathbb{Z}$, which we call alternate knot contact homology; the alternate theory lacks some of the properties of the usual theory but produces a knot invariant which seems to contain more information.

The paper is organized as follows. In Section 2, we define the braid and knot DGAs and state the main invariance results. Section 3 gives a highly informal description of how our invariants relate to contact geometry. Section 4 defines 
contact homology for braids and knots and proves invariance of $H C_{0}$; we subsequently return to the invariance proof of the full DGAs in Section 5 Section 6 presents properties of the invariants, including augmentation numbers and behavior under mirrors, and Section [7 relates knot contact homology to the knot's determinant. Section 8 gives some examples of computations of the invariants. We discuss the alternate knot invariant in Section 9

In the sequel to this paper [19], we give a geometric interpretation of $\phi$ via the usual treatment of $B_{n}$ as a mapping class group. We then use this to present a new, completely topological definition for $H C_{0}$ for braids and knots, in terms of cords and relations similar to skein relations, and find a relation to character varieties. This interpretation explains, independent of contact geometry, "why" $H C_{0}$ is a topological invariant.

Acknowledgements I am grateful to Yasha Eliashberg for suggesting the project and the means to approach it and offering a great deal of insightful guidance. I would also like to thank John Etnyre, Michael Hutchings, Kiran Kedlaya, Tom Mrowka, Josh Sabloff, Ravi Vakil, and Ke Zhu for useful conversations, and the Institute for Advanced Study, the American Institute of Mathematics, and Stanford University for their hospitality. Funding was provided by a Five-Year Fellowship from the American Institute of Mathematics, and at one point by NSF grant DMS-9729992.

\section{Definitions and main results}

\subsection{Differential graded algebras}

In contact geometry, the homology of a certain DGA defines the relative contact homology of a Legendrian submanifold in a contact manifold. In all known cases, a Legendrian isotopy invariant is provided not only by relative contact homology, but also by the stable tame isomorphism class of the DGA.

We recall the basic definitions, originally due to Chekanov $\left[3\right.$. Let $\mathbb{Z}\left\langle a_{1}, \ldots, a_{n}\right\rangle$ denote the (noncommutative, unital) tensor algebra generated by $a_{1}, \ldots, a_{n}$, which is generated as a $\mathbb{Z}$-module by words in the $a_{i}$ 's, including the empty word.

Definition 2.1 Equip $\mathcal{A}=\mathbb{Z}\left\langle a_{1}, \ldots, a_{n}\right\rangle$ with a grading over $\mathbb{Z}$ by assigning degrees to the generators $a_{i}$. Then a differential on $\mathcal{A}=\mathbb{Z}\left\langle a_{1}, \ldots, a_{n}\right\rangle$ is a map $\partial: \mathcal{A} \rightarrow \mathcal{A}$ which lowers degree by 1 and satisfies $\partial^{2}=0$ and the Leibniz rule 
$\partial(v w)=(\partial v) w+(-1)^{\operatorname{deg} v} v(\partial w)$. The pair $(\mathcal{A}, \partial)$ is what we call a differential graded algebra, for the purposes of this paper.

To define a particular equivalence relation between DGAs known as stable tame isomorphism, we need several more notions, beginning with a refinement of the usual notion of isomorphism.

Definition 2.2 An algebra map $\phi:\left(\mathbb{Z}\left\langle a_{1}, \ldots, a_{n}\right\rangle, \partial\right) \rightarrow\left(\mathbb{Z}\left\langle\tilde{a}_{1}, \ldots, \tilde{a}_{n}\right\rangle, \tilde{\partial}\right)$ is an elementary isomorphism if it is a graded chain map and, for some $i$,

$$
\phi\left(a_{j}\right)= \begin{cases} \pm \tilde{a}_{i}+v & \text { for } j=i \text { and some } v \in \mathbb{Z}\left\langle\tilde{a}_{1}, \ldots, \tilde{a}_{i-1}, \tilde{a}_{i+1}, \ldots, \tilde{a}_{n}\right\rangle \\ \tilde{a}_{j} & \text { for } j \neq i .\end{cases}
$$

A tame isomorphism between DGAs is any composition of elementary isomorphisms.

In the above definition, the order of the generators is immaterial; that is, a map which simply permutes the generators of the DGA (and treats the grading and differential accordingly) is considered to be elementary. Note also that elementary and tame isomorphisms are in fact isomorphisms.

A "trivial" example of a DGA is given by $\left(\mathcal{E}^{i}=\mathbb{Z}\left\langle e_{1}^{i}, e_{2}^{i}\right\rangle, \partial\right)$, where $\operatorname{deg} e_{1}^{i}-1=$ $\operatorname{deg} e_{2}^{i}=i$ and $\partial e_{1}^{i}=e_{2}^{i}, \partial e_{2}^{i}=0$. The main equivalence relation between DGAs, which we now present, stipulates that adding this trivial DGA does not change equivalence class.

Definition 2.3 The degree- $i$ algebraic stabilization of the DGA $\left(\mathcal{A}=\mathbb{Z}\left\langle a_{1}\right.\right.$, $\left.\left.\ldots, a_{n}\right\rangle, \partial\right)$ is the DGA $\left(S^{i}(\mathcal{A})=\mathbb{Z}\left\langle a_{1}, \ldots, a_{n}, e_{1}^{i}, e_{2}^{i}\right\rangle, \partial\right)$, with grading and differential induced from $\mathcal{A}$ and $\mathcal{E}^{i}$. Two DGAs $(\mathcal{A}, \partial)$ and $(\tilde{\mathcal{A}}, \tilde{\partial})$ are stable tame isomorphic if there exist algebraic stabilizations $\left(S^{i_{1}}\left(\cdots\left(S^{i_{k}}(\mathcal{A})\right) \cdots\right), \partial\right)$ and $\left(S^{\tilde{i}_{1}}\left(\cdots\left(S_{\tilde{i}_{\tilde{k}}}(\tilde{\mathcal{A}})\right) \cdots\right), \tilde{\partial}\right)$ which are tamely isomorphic.

Stable tame isomorphism is designed to be a special case of quasi-isomorphism.

Proposition 2.4 If $(\mathcal{A}, \partial)$ and $(\tilde{\mathcal{A}}, \tilde{\partial})$ are stable tame isomorphic, then the homologies $H_{*}(\mathcal{A}, \partial)$ and $H_{*}(\tilde{\mathcal{A}}, \tilde{\partial})$ are isomorphic.

The proof of Proposition 2.4 can be found, eg, in 9 . 


\subsection{The invariants}

We now introduce the knot and braid invariants. The definition of the knot DGA depends on the braid definition, which we give first.

Let $B_{n}$ denote the braid group on $n$ strands, which is generated by $\sigma_{1}, \ldots, \sigma_{n-1}$, with relations $\sigma_{i} \sigma_{i+1} \sigma_{i}=\sigma_{i+1} \sigma_{i} \sigma_{i+1}$ for $1 \leq i \leq n-1$, and $\sigma_{i} \sigma_{j}=\sigma_{j} \sigma_{i}$ for $|i-j| \geq 2$. Write $\mathcal{A}_{n}$ for the tensor algebra on $n(n-1)$ generators which we label as $a_{i j}$ for $1 \leq i, j \leq n$ and $i \neq j$, and let $\operatorname{Aut}\left(\mathcal{A}_{n}\right)$ denote the group of algebra automorphisms of $\mathcal{A}_{n}$.

For each generator $\sigma_{k}$ of $B_{n}$, define the tame automorphism $\phi_{\sigma_{k}} \in \operatorname{Aut}\left(\mathcal{A}_{n}\right)$ by its action on the generators of $\mathcal{A}_{n}$ :

$$
\phi_{\sigma_{k}}:\left\{\begin{array}{rlrl}
a_{k i} & \mapsto-a_{k+1, i}-a_{k+1, k} a_{k i} & & i \neq k, k+1 \\
a_{i k} & \mapsto-a_{i, k+1}-a_{i k} a_{k, k+1} & i \neq k, k+1 \\
a_{k+1, i} & \mapsto a_{k i} & i \neq k, k+1 \\
a_{i, k+1} & \mapsto a_{i k} & i \neq k, k+1 \\
a_{k, k+1} & \mapsto a_{k+1, k} & \\
a_{k+1, k} & \mapsto a_{k, k+1} & i, j \neq k, k+1 . \\
a_{i j} & \mapsto a_{i j} &
\end{array}\right.
$$

Proposition $2.5 \phi$ extends to a homomorphism from $B_{n}$ to $\operatorname{Aut}\left(\mathcal{A}_{n}\right)$.

Proof The fact that $\phi$ preserves the braid relations in $B_{n}$ is a direct computation.

We will denote the image of $B \in B_{n}$ under $\phi$ as $\phi_{B} \in \operatorname{Aut}\left(\mathcal{A}_{n}\right)$.

This representation was essentially first studied by Magnus [16] in the context of automorphisms of free groups; more precisely, he presented a representation on a polynomial algebra in $\left(\begin{array}{l}n \\ 2\end{array}\right)$ variables, which we can derive from $\phi$ by abelianizing and setting $a_{i j}=a_{j i}$ for all $i, j$. Humphries [12] subsequently extended the representation to a polynomial algebra in $n(n-1)$ variables, and interpreted it in terms of transvections. Our homomorphism is simply the lift of Humphries' representation to the corresponding tensor algebra. We note that the starting point in [19] is a new topological interpretation of $\phi$ in terms of skein relations.

We can obtain a linear representation from $\phi$ by finding a finite-dimensional piece of $\mathcal{A}_{n}$ on which $\phi$ descends; in the language of [8], this involves linearizing $\phi$ with respect to some augmentation. Concretely, if we write $\mathcal{M}_{n}$ as the subalgebra of $\mathcal{A}_{n}$ generated by $\left\{a_{i j}+2\right\}$, then $\phi$ descends to $\mathcal{M}_{n} / \mathcal{M}_{n}^{2}$. If we further 
quotient by setting $a_{i j}=a_{j i}$ for all $i, j$, then we obtain an $\left(\begin{array}{l}n \\ 2\end{array}\right)$-dimensional representation of $B_{n}$, which is precisely the inverse of the Lawrence-Krammer representation [14 with $q=-1$ and $t=1$. Indeed, there is a striking similarity in appearance between $\phi$ and the Lawrence-Krammer representation which we further explore in [19].

Unlike Lawrence-Krammer, $\phi$ does not give a faithful representation of $B_{n}$; this is not overly surprising, since $B_{n}$ has nontrivial center, while $\operatorname{Aut}\left(\mathcal{A}_{n}\right)$ does not. Indeed, a result from [12] states that the kernel of $\phi$ is precisely the center of $B_{n}$, which is generated by $\left(\sigma_{1} \cdots \sigma_{n-1}\right)^{n}$. However, the extension map $\phi^{\text {ext }}: B_{n} \hookrightarrow B_{n+1} \stackrel{\phi}{\rightarrow} \operatorname{Aut}\left(\mathcal{A}_{n+1}\right)$ is faithful, a fact first established algebraically in [16. We will return to $\phi^{\text {ext }}$ in a moment; geometric proofs of the faithfulness results can be found in [19.

We now define the DGA invariant for braids.

Definition 2.6 Let $B \in B_{n}$ be a braid. Let $\mathcal{A}$ be the graded tensor algebra on $2 n(n-1)$ generators, $\left\{a_{i j} \mid 1 \leq i, j \leq n, i \neq j\right\}$ of degree 0 and $\left\{b_{i j} \mid 1 \leq\right.$ $i, j \leq n, i \neq j\}$ of degree 1 . Define the differential $\partial$ on $\mathcal{A}$ by

$$
\partial b_{i j}=a_{i j}-\phi_{B}\left(a_{i j}\right), \quad \partial a_{i j}=0 .
$$

Then we call $(\mathcal{A}, \partial)$ the braid $D G A$ of $B$.

Theorem 2.7 If $B, \tilde{B}$ are conjugate in $B_{n}$, then their braid DGAs are stable tame isomorphic.

We defer the proof of Theorem 2.7 until Section [5.1. The DGA invariant for knots is also derived from the map $\phi$; in fact, we will see in Section 4.2 that it contains the braid DGA. Before introducing it, we need some more notation.

As mentioned before, we can define a map $\phi^{\text {ext }}: B_{n} \rightarrow \operatorname{Aut}\left(\mathcal{A}_{n+1}\right)$ induced from $\phi$ through the inclusion $B_{n} \hookrightarrow B_{n+1}$ obtained by adding an $(n+1)$-st strand which does not interact with the other $n$ strands; that is, each generator $\sigma_{i} \in B_{n}$ is mapped to $\sigma_{i} \in B_{n+1}$ for $1 \leq i \leq n-1$. When dealing with $\phi^{\text {ext }}$, we replace all indices $(n+1)$ by an asterisk; ie, write $a_{* i}, a_{i *}$ for $a_{n+1, i}, a_{i, n+1}$, respectively. Since the $(n+1)$-st strand does not interact with the other strands, it follows from the definition of $\phi$ that for $B \in B_{n}, \phi_{B}^{\operatorname{ext}}\left(a_{* i}\right)$ is a linear combination of $a_{* j}, 1 \leq j \leq n$, with coefficients in $\mathcal{A}_{n}$, and similarly for $\phi_{B}^{\text {ext }}\left(a_{i *}\right)$.

Definition 2.8 The $n \times n$ matrices $\Phi_{B}^{L}(A)$ and $\Phi_{B}^{R}(A)$ are defined by

$$
\phi_{B}^{\mathrm{ext}}\left(a_{i *}\right)=\sum_{j=1}^{n}\left(\Phi_{B}^{L}(A)\right)_{i j} a_{j *} \quad \text { and } \quad \phi_{B}^{\mathrm{ext}}\left(a_{* j}\right)=\sum_{i=1}^{n} a_{* i}\left(\Phi_{B}^{R}(A)\right)_{i j} .
$$


The matrices $\Phi_{B}^{L}(A)$ and $\Phi_{B}^{R}(A)$ can be obtained from each other by the "conjugation" operation discussed in Section 6.1. Though $\Phi_{B}^{L}(A), \Phi_{B}^{R}(A)$ are derived from $\phi$, they also determine $\phi$; see Proposition 6.2 .

To define the DGA for knots, it is convenient to assemble generators of the DGA into matrices. For notational purposes, let $A$ denote the matrix $\left(a_{i j}\right)$, where we set $a_{i i}=-2$ for all $i$, and similarly write $B=\left(b_{i j}\right), C=\left(c_{i j}\right)$, $D=\left(d_{i j}\right)$, where $b_{i j}, c_{i j}, d_{i j}$ are variables used below with no stipulation on $b_{i i}, c_{i i}, d_{i i}$; also, write $\partial A$ for the matrix $\left(\partial a_{i j}\right), \phi_{B}(A)$ for $\left(\phi_{B}\left(a_{i j}\right)\right)$, and so forth. (There should hopefully be no confusion between a braid $B$ and the matrix $B$.) We will sometimes view $A$ as a matrix of variables, so that, eg, we define $\Phi_{B}^{L}(M)$ for an $n \times n$ matrix $M$ to be the evaluation of $\Phi_{B}^{L}(A)$ when we set $a_{i j}=M_{i j}$ for all $i, j$. (Note that this requires $M_{i i}=-2$ for all $i$.)

Definition 2.9 Let $B \in B_{n}$. Write $\mathcal{A}$ for the tensor algebra with the following generators: $\left\{a_{i j} \mid 1 \leq i, j \leq n, i \neq j\right\}$ of degree $0 ;\left\{b_{i j}, c_{i j} \mid 1 \leq i, j \leq n\right\}$ of degree 1 ; and $\left\{d_{i j} \mid 1 \leq i, j \leq n\right\}$ and $\left\{e_{i} \mid 1 \leq i \leq n\right\}$ of degree 2. Define the differential $\partial$ on $\mathcal{A}$ by

$$
\begin{aligned}
\partial A & =0 \\
\partial B & =\left(1-\Phi_{B}^{L}(A)\right) \cdot A \\
\partial C & =A \cdot\left(1-\Phi_{B}^{R}(A)\right) \\
\partial D & =B \cdot\left(1-\Phi_{B}^{R}(A)\right)-\left(1-\Phi_{B}^{L}(A)\right) \cdot C \\
\partial e_{i} & =\left(B+\Phi_{B}^{L}(A) \cdot C\right)_{i i},
\end{aligned}
$$

where - denotes matrix multiplication. Then we call $(\mathcal{A}, \partial)$ the knot DGA of $B$.

The fact that $\partial^{2}=0$ in the knot DGA is not obvious; while it is clear that $\partial^{2} A=\partial^{2} B=\partial^{2} C=\partial^{2} D=0$, we have $\partial^{2} e_{i}=-2-\left(\Phi_{B}^{L}(A) \cdot A \cdot \Phi_{B}^{R}(A)\right)_{i i}$, and we need Proposition 4.7 to conclude that $\partial^{2} e_{i}=0$. The key result for knot DGAs is the following theorem, which allows the knot DGA to descend from braids to knots.

Theorem 2.10 If the closures of two braids $B, \tilde{B}$ are the same knot, then the knot DGAs for $B, \tilde{B}$ are stable tame isomorphic.

The proof of Theorem [2.10] is postponed until Section [5.2] after we first introduce $H C_{0}$ and present the easier proof of invariance for $H C_{0}$.

By Alexander's Theorem, every knot in $\mathbb{R}^{3}$ can be represented as a closed braid. Theorem 2.10 implies that the following notion is well-defined. 
Definition 2.11 Let $K \subset \mathbb{R}^{3}$ be a knot. The knot DGA class of $K$ is the equivalence class (under stable tame isomorphism) of the knot DGA of any braid whose closure is $K$.

We will occasionally abuse notation and speak of the "knot DGA" of $K$; this is understood to be modulo equivalence. The knot DGA can equally well be defined for links, for which it also gives an invariant.

We conclude this section by explicitly computing, as an example, the knot DGA for the trefoil, which is the closure of $\sigma_{1}^{3} \in B_{2}$. By the definition of $\phi$, we see that $\phi_{\sigma_{1}}^{\text {ext }}$ acts as follows: $a_{12} \mapsto a_{21}, a_{21} \mapsto a_{12}, a_{1 *} \mapsto-a_{2 *}-a_{21} a_{1 *}$, $a_{2 *} \mapsto a_{1 *}, a_{* 1} \mapsto-a_{* 2}-a_{* 1} a_{12}, a_{* 2} \mapsto a_{* 1}$. Iterating $\phi_{\sigma_{1}}^{\text {ext }}$ three times on $a_{1 *}, a_{2 *}, a_{* 1}, a_{* 2}$ yields

and

$$
\begin{gathered}
\Phi_{\sigma_{1}^{3}}^{L}(A)=\left(\begin{array}{cc}
2 a_{21}-a_{21} a_{12} a_{21} & 1-a_{21} a_{12} \\
-1+a_{12} a_{21} & a_{12}
\end{array}\right) \\
\Phi_{\sigma_{1}^{3}}^{R}(A)=\left(\begin{array}{cc}
2 a_{12}-a_{12} a_{21} a_{12} & -1+a_{12} a_{21} \\
1-a_{21} a_{12} & a_{21}
\end{array}\right) .
\end{gathered}
$$

One can now calculate the differential on the knot DGA of $\sigma_{1}^{3}$ :

$$
\begin{aligned}
& \partial b_{11}=-2+3 a_{21}-a_{21} a_{12} a_{21} \\
& \partial c_{11}=-2+3 a_{12}-a_{12} a_{21} a_{12} \\
& \partial b_{12}= 2+a_{12}-4 a_{21} a_{12}+a_{21} a_{12} a_{21} a_{12} \\
& \partial c_{21}= 2+a_{21}-4 a_{21} a_{12}+a_{21} a_{12} a_{21} a_{12} \\
& \partial b_{21}=-2+a_{21}+a_{12} a_{21} \\
& \partial c_{12}=-2+a_{12}+a_{12} a_{21} \\
& \partial b_{22}=-2+3 a_{12}-a_{12} a_{21} a_{12} \\
& \partial c_{22}=-2+3 a_{21}-a_{21} a_{12} a_{21} \\
& \partial d_{11}= b_{11}-b_{12}-c_{11}+c_{21}+2 a_{21} c_{11}-2 b_{11} a_{12}-a_{21} a_{12} c_{21}+b_{12} a_{21} a_{12} \\
& \quad \quad-a_{21} a_{12} a_{21} c_{11}+b_{11} a_{12} a_{21} a_{12} \\
& \quad \quad-b_{11} a_{12} a_{21}-a_{21} a_{12} a_{21} c_{12} \\
& \partial d_{12}=b_{11}+b_{12}-c_{12}+c_{22}+2 a_{21} c_{12}-b_{12} a_{21}-a_{21} a_{12} c_{22} \\
& \quad \quad+b_{22} a_{21} a_{12}+b_{21} a_{12} a_{21} a_{12} \\
& \partial d_{21}=b_{21}-b_{22}-c_{11}-c_{21}+a_{12} c_{21}-2 b_{21} a_{12}+a_{12} a_{21} c_{11} \\
& \partial d_{22}=b_{21}+b_{22}-c_{12}-c_{22}+a_{12} c_{22}-b_{22} a_{21}+a_{12} a_{21} c_{12}-b_{21} a_{12} a_{21} \\
& \partial e_{1}=b_{11}+c_{21}+2 a_{21} c_{11}-a_{21} a_{12} c_{21}-a_{21} a_{12} a_{21} c_{11} \\
& \partial e_{2}=b_{22}-c_{12}+a_{12} c_{22}+a_{12} a_{21} c_{12} .
\end{aligned}
$$

We will return to this example in future sections. 


\section{Motivation from contact geometry}

Having defined the knot and braid DGAs in the previous section, we now describe the background in contact geometry leading to the development of these invariants. It should be noted that work is in progress to establish rigorously that the DGAs give the relative contact homology theory which we describe below.

Given a smooth manifold $M$, the cotangent bundle $T^{*} M$ has a canonical symplectic structure given by $\omega=d \lambda$, where $\lambda$ is the tautological one-form on $T^{*} M$ sending a tangent vector in $T^{*} M$ to the pairing between the base point in $T^{*} M$ and the projected tangent vector in $T M$ under the projection $\pi: T^{*} M \rightarrow M$. For any smooth submanifold $N \subset M$, the conormal bundle $\mathcal{L} N=\left\{\theta \in T^{*} M \mid\langle\theta, v\rangle=0\right.$ for all $\left.v \in T_{\pi(v)} N\right\}$ over $N$ is then a Lagrangian submanifold of $T^{*} M$, and smooth isotopy of $N$ leads to Lagrangian isotopy of $\mathcal{L} N$. This setup (with $N$ a knot) was introduced in the physics literature in [20], and was communicated to the author in this generality by Eliashberg.

Partly because $\mathcal{L} N$ is noncompact, it is more convenient for us to consider a slightly modified setup. Suppose that $M$ is equipped with a metric. Then the cosphere bundle $S T^{*} M$ of unit covectors in $T^{*} M$ ("co-oriented contact elements in $M$ ") has a canonical contact structure induced by the one-form $\lambda$. If $N \subset M$ is a compact smooth submanifold, then the unit conormal bundle $L N=\mathcal{L} N \cap S T^{*} M$ is a compact Legendrian submanifold of $S T^{*} M$, and smooth isotopy of $N$ leads to Legendrian isotopy of $L N$.

In recent years, beginning with work of Eliashberg and Hofer, invariants of Legendrian isotopy in contact manifolds have been developed, via holomorphiccurve techniques, using relative contact homology [6] and the more general Symplectic Field Theory [7]. In particular, for Legendrian knots in $\mathbb{R}^{3}$ with the standard contact structure, Chekanov [3] developed a purely combinatorial formulation of relative contact homology; this work was subsequently extended in 9, 18. Relative contact homology was also studied for knots in circle bundles in [23], and for $\mathbb{R}^{2 n+1}$ with the standard contact structure in [5]. In all of these special cases, relative contact homology is given by the homology of a certain DGA whose stable tame isomorphism class is an invariant. With the exception of [5], however, the DGAs are defined combinatorially rather than geometrically, with invariance proofs given by combinatorics as well. The invariants in the present paper are likewise combinatorial in nature.

We now specialize, as in [20], to the case when $M=\mathbb{R}^{3}$ and $K \subset \mathbb{R}^{3}$ is a knot. Then $L K$ is an embedded Legendrian 2-torus in the 5 -dimensional 
contact manifold $S T^{*} \mathbb{R}^{3}=\mathbb{R}^{3} \times S^{2}$, which is contactomorphic to the 1 -jet space $J^{1}\left(S^{2}\right)$ with the standard contact structure. We note that $L K$ is always topologically unknotted since it has codimension 3 in $S T^{*} \mathbb{R}^{3}$; however, its Legendrian isotopy type yields information about the knot $K$. In fact, the knot DGA defined in Section 2.2. which is a nontrivial invariant, conjecturally gives the relative contact homology of $L K$ in $S T^{*} \mathbb{R}^{3}$.

A local model suggested by Eliashberg allows us to study conjugacy classes of braids as a first step. Let $K \subset \mathbb{R}^{3}$ be any knot (or, in particular, the unknot), and let $B$ be a braid. A sufficiently small neighborhood of $L K$ is contactomorphic to $J^{1}(L K)=J^{1}\left(T^{2}\right)$. If we glue $B$ along a tubular neighborhood of $K$ to produce a new knot $\tilde{K}$, then $L \tilde{K}$ is in an arbitrarily small neighborhood of $L K$, and thus gives a Legendrian 2-torus in $J^{1}\left(T^{2}\right)$. A smooth isotopy of $B$, or a conjugation operation on $B$, yields a Legendrian isotopy of this torus in $J^{1}\left(T^{2}\right)$. Then the braid DGA from Section 2.2 conjecturally gives the relative contact homology of $L \tilde{K}$ in $J^{1}\left(T^{2}\right)$.

Recall from, eg, 6] that the relative contact homology for a Legendrian submanifold $L$ of a contact manifold $(V, \alpha)$ is the homology of a DGA whose generators are Reeb chords of $L$, ie, flow lines of the Reeb vector field which begin and end on $L$. The differential is then defined by counting certain holomorphic maps of punctured disks into the symplectization $V \times \mathbb{R}$ of $V$, with boundary on $L \times \mathbb{R}$ and punctures limiting to Reeb chords.

For the case of a knot $K$ in $\mathbb{R}^{3}$, the contact manifold is $\mathbb{R}^{3} \times S^{2}$, with Reeb vector field pointing in the $\mathbb{R}^{3}$ fibers, in the direction specified by the underlying point in $S^{2}$. It follows that Reeb chords for $L K$ correspond to "binormal chords" to $K$ in $\mathbb{R}^{3}$, ie, oriented line segments in $\mathbb{R}^{3}$ with endpoints on $K$ which are normal to $K$ at both endpoints. For instance, the unknot given by an ellipse in the plane yields four Reeb chords, corresponding to the major and minor axes traversed either way.

If $K$ is the closure of a braid $B \in B_{n}$, we can embed $B$ in a neighborhood of an elliptical unknot in such a way that the Reeb chords for $L K$ come in two families:

- $2 n(n-1)$ "small chords" within the neighborhood of the unknot, four for each pair of braid strands; these divide further into two Reeb chords where the pair of strands is closest $\left(a_{i j}\right)$ and two where the pair is farthest $\left(b_{i j}\right)$

- $4 n^{2}$ "big chords" corresponding to the Reeb chords of the ellipse; each binormal chord of the ellipse yields $n^{2}$ binormal chords for $K\left(c_{i j}, d_{i j}\right.$ for 
the minor-axis chords, $e_{i j}, f_{i j}$ for the major-axis chords), since there are $n$ choices for each endpoint.

For the braid itself, the Reeb chords for $L B \subset J^{1}\left(T^{2}\right)$ correspond to the $2 n(n-$ 1) "small chords" $a_{i j}, b_{i j}$.

This gives a rough explanation for the generators of the knot and braid DGAs; for the knot case, this actually gives the modified knot DGA defined below in Proposition 4.8. Showing that the differentials of these DGAs actually count the appropriate holomorphic disks is beyond the scope of this paper. The analytical details, which use an approach due to Fukaya and Oh [10 of counting gradient flow trees, are the subject of work in progress. As in the theory of Legendrian knots in standard contact $\mathbb{R}^{3}$, however, the combinatorial proof of the invariance of our DGAs under isotopy gives evidence for the validity of our "computation" of relative contact homology.

\section{Knot and braid contact homology}

In this section, we examine the homology of the DGAs defined in Section 2, and focus on a particular piece for which isotopy invariance is easy to establish. We will return to invariance proofs of the full DGAs in Section [5]

\subsection{Definitions}

In order to use the knot and braid DGAs as invariants, we need to know when two DGAs are stable tame isomorphic. This can be done through "computable" invariants of equivalence classes of DGAs, including Poincaré polynomials 3 and the characteristic algebra [18.

In the case of braid and knot DGAs, it turns out that the first order Poincaré polynomial over $\mathbb{Z}_{2}$ corresponding to the trivial augmentation gives no interesting invariant of the braid conjugacy class or the knot. (See however Section 8.3 for a different result for a nontrivial augmentation over $\mathbb{Z}_{2}$, and Section $\mathbf{7 . 2}$ for linearization over $\mathbb{Z}$.) This is because to first order over $\mathbb{Z}_{2}$, the homomorphism $\phi$ factors through the projection $B_{n} \rightarrow S_{n}$ to the symmetric group. However, the degree 0 part of the characteristic algebra already yields interesting invariants; we recall from [18] that the characteristic algebra of a DGA $(\mathcal{A}, \partial)$ is the quotient of $\mathcal{A}$ by the two-sided ideal generated by im $\partial$. Before proceeding further, we define the contact homology of a braid or knot. 
Definition 4.1 The contact homology of a braid $B$, written $H C_{*}(B)$, is defined to be the (graded) homology of the braid DGA of $B$. The contact homology of a knot $K$, written $H C_{*}(K)$, is the (graded) homology of the knot DGA of any braid whose closure is $K$. More generally, if $R$ is any ring, then we define as usual $H C_{*}(B ; R)$ and $H C_{*}(K ; R)$ to be the homology of the appropriate DGA tensored with $R$.

Proposition 4.2 The degree 0 contact homology of knots and braids is the degree 0 part of the characteristic algebra of the corresponding DGA. More explicitly, for $B \in B_{n}$, let $\mathcal{I}_{B}^{\text {braid }}$ be the two-sided ideal of $\mathcal{A}_{n}$ generated by $\left\{a_{i j}-\phi_{B}\left(a_{i j}\right)\right\}$, while $\mathcal{I}_{B}^{\mathrm{knot}}$ is the two-sided ideal of $\mathcal{A}_{n}$ generated by the entries of the two matrices $\left(1-\Phi_{B}^{L}(A)\right) \cdot A$ and $A \cdot\left(1-\Phi_{B}^{R}(A)\right)$. Then $H C_{0}(B)=$ $\mathcal{A}_{n} / \mathcal{I}_{B}^{\text {braid }}$ and $H C_{0}(K)=\mathcal{A}_{n} / \mathcal{I}_{B}^{\mathrm{knot}}$, where $K$ is the closure of $B$.

Proof Since the braid and knot DGAs of $B \in B_{n}$ have no generators in negative degree, the entirety of $\mathcal{A}_{n}$ (which is the degree 0 part of the algebra) consists of cycles. On the other hand, the image of $\partial$ in degree 0 is precisely the ideal generated by the images under $\partial$ of the generators in degree 1 , and so the ideal of boundaries in degree 0 is given by $\mathcal{I}_{B}^{\text {braid }}, \mathcal{I}_{B}^{\text {knot }}$ for the braid and knot DGA, respectively.

For example, for the trefoil, $H C_{0}\left(3_{1}\right)$ is given by $\mathbb{Z}\left\langle a_{12}, a_{21}\right\rangle$ modulo the relations provided by $\partial b_{i j}, \partial c_{i j}$ as calculated in Section 2.2. From $\partial b_{21}$ and $\partial c_{12}$, we have relations $-2+a_{21}+a_{12} a_{21}=-2+a_{12}+a_{12} a_{21}=0$, and hence $a_{12}=a_{21}$. If we set $a_{12}=a_{21}=x$, then the $\partial b_{i j}, \partial c_{i j}$ relations become $\left\{x^{3}-3 x+2, x^{4}-4 x^{2}+x+2, x^{2}+x-2\right\}$. The gcd of these polynomials is $x^{2}+x-2$, and so $H C_{0}\left(3_{1}\right) \cong \mathbb{Z}[x] /\left(x^{2}+x-2\right)$.

In practice, we will study $H C_{0}$ rather than the full braid and knot DGAs, because it is relatively easy to compute, as the trefoil example demonstrates. It is immediate from Proposition 2.4 and Theorems 2.6 and 2.9 that $H C_{0}$ gives an invariant of braid group conjugacy classes and knots. However, there is a direct proof of the invariance of $H C_{0}$ which is much simpler notationally than the proofs of Theorems 2.6 and 2.9 while containing the main ideas from these proofs. We will give this direct proof below for braids, and in Section 4.3 for knots.

Theorem 4.3 Up to isomorphism, $H C_{0}(B)$ is an invariant of the conjugacy class of the braid $B$. 
Proof Suppose that $B, \tilde{B}$ are conjugate in the braid group $B_{n}$, so that $\tilde{B}=$ $C^{-1} B C$ for some $C \in B_{n}$. We use the notation of Proposition 4.2 .

First note that $\mathcal{I}_{B}^{\text {braid }} \subset \mathcal{A}_{n}$ is the ideal generated by the image of the map $1-\phi_{B}: \mathcal{A}_{n} \rightarrow \mathcal{A}_{n}:$ if $x_{1}, x_{2} \in \mathcal{A}_{n}$, then $\left(1-\phi_{B}\right)\left(x_{1} x_{2}\right)=\left(1-\phi_{B}\right)\left(x_{1}\right) x_{2}+$ $\phi_{B}\left(x_{1}\right)\left(1-\phi_{B}\right)\left(x_{2}\right)$ is in the ideal generated by $\left(1-\phi_{B}\right)\left(x_{1}\right)$ and $\left(1-\phi_{B}\right)\left(x_{2}\right)$. Similarly, $\mathcal{I}_{\tilde{B}}^{\text {braid }}$ is the ideal generated by the image of $1-\phi_{\tilde{B}}=1-\phi_{C}^{-1} \phi_{B} \phi_{C}$. It follows by inspection of the commutative diagram

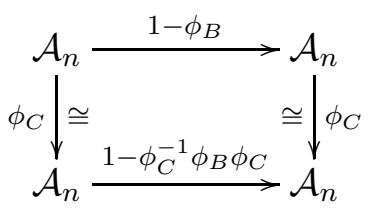

that $\mathcal{I}_{\tilde{B}}^{\text {braid }}$ is the image of $\mathcal{I}_{B}^{\text {braid }}$ under the automorphism $\phi_{C}$ on $\mathcal{A}_{n}$, and hence that $H C_{0}(B)=\mathcal{A}_{n} / \mathcal{I}_{B}^{\text {braid }}$ and $H C_{0}(\tilde{B})=\mathcal{A}_{n} / \mathcal{I}_{\tilde{B}}^{\text {braid }}$ are isomorphic.

The corresponding invariance result for knots requires a few preparatory results, which are interesting in their own right; we give these in the following section, and prove $H C_{0}$ invariance for knots in Section 4.3

\subsection{Ancillary results}

Here we collect a number of results about $\phi, \Phi^{L}$, and $\Phi^{R}$ which will be crucial for future sections.

Proposition 4.4 (Chain Rule) If $B_{1}, B_{2} \in B_{n}$, then

$\Phi_{B_{1} B_{2}}^{L}(A)=\Phi_{B_{2}}^{L}\left(\phi_{B_{1}}(A)\right) \cdot \Phi_{B_{1}}^{L}(A) \quad$ and $\quad \Phi_{B_{1} B_{2}}^{R}(A)=\Phi_{B_{1}}^{R}(A) \cdot \Phi_{B_{2}}^{R}\left(\phi_{B_{1}}(A)\right)$.

Proof We will establish the result for $\Phi^{L}$; the proof for $\Phi^{R}$ is completely analogous. By the definition of $\Phi^{L}$, we have

$$
\phi_{B_{1} B_{2}}^{\mathrm{ext}}\left(a_{i *}\right)=\sum_{j=1}^{n}\left(\Phi_{B_{1} B_{2}}^{L}(A)\right)_{i j} a_{j *} ;
$$

on the other hand,

$$
\begin{aligned}
\phi_{B_{1} B_{2}}^{\text {ext }}\left(a_{i *}\right) & =\phi_{B_{1}}^{\text {ext }} \phi_{B_{2}}^{\text {ext }}\left(a_{i *}\right) \\
& =\sum_{j=1}^{n} \phi_{B_{1}}^{\operatorname{ext}}\left(\left(\Phi_{B_{2}}^{L}(A)\right)_{i j} a_{j *}\right)
\end{aligned}
$$




$$
\begin{aligned}
& =\sum_{j=1}^{n}\left(\Phi_{B_{2}}^{L}\left(\phi_{B_{1}}(A)\right)\right)_{i j} \phi_{B_{1}}^{\mathrm{ext}}\left(a_{j *}\right) \\
& =\sum_{j, \ell=1}^{n}\left(\Phi_{B_{2}}^{L}\left(\phi_{B_{1}}(A)\right)\right)_{i j}\left(\Phi_{B_{1}}^{L}(A)\right)_{j \ell} a_{\ell *},
\end{aligned}
$$

and the result follows.

A word of explanation is in order for the penultimate equality in the chain above, because it may seem backwards. Applying $\phi_{B_{1}}^{\text {ext }}$ to $\left(\Phi_{B_{2}}^{L}(A)\right)_{i j} a_{j *}$ entails replacing each $a_{i j}$ by $\phi_{B_{1}}\left(a_{i j}\right)$ and each $a_{j *}$ by $\phi_{B_{1}}^{\text {ext }}\left(a_{j *}\right)$, and the result is $\left(\Phi_{B_{2}}^{L}\left(\phi_{B_{1}}(A)\right)\right)_{i j} \phi_{B_{1}}^{\text {ext }}\left(a_{j *}\right)$.

Corollary 4.5 For any braid $B$,

$$
\Phi_{B^{-1}}^{L}\left(\phi_{B}(A)\right)=\left(\Phi_{B}^{L}(A)\right)^{-1} \quad \text { and } \quad \Phi_{B^{-1}}^{R}\left(\phi_{B}(A)\right)=\left(\Phi_{B}^{R}(A)\right)^{-1} .
$$

Proof Set $B_{1}=B, B_{2}=B^{-1}$ in Proposition 4.4 and use the fact that $\Phi_{\mathrm{id}}^{L}(A)=\Phi_{\mathrm{id}}^{R}(A)=1$.

Lemma 4.6 The matrices $\Phi_{\sigma_{k}}^{L}(A)$ and $\Phi_{\sigma_{k}}^{R}(A)$ are identical to the $n \times n$ identity matrix, outside of the $2 \times 2$ submatrix formed by rows $k, k+1$ and columns $k, k+1$, which is given by $\left(\begin{array}{cc}-a_{k+1, k} & -1 \\ 1 & 0\end{array}\right)$ for $\Phi_{\sigma_{k}}^{L}(A)$ and $\left(\begin{array}{cc}-a_{k, k+1} & 1 \\ -1 & 0\end{array}\right)$ for $\Phi_{\sigma_{k}}^{R}(A)$.

Proof This is immediate from the definitions of $\phi, \Phi^{L}$, and $\Phi^{R}$.

Proposition 4.7 For any braid $B, \phi_{B}(A)=\Phi_{B}^{L}(A) \cdot A \cdot \Phi_{B}^{R}(A)$.

Proof When $B=\sigma_{k}$ for some $k$, the identity can be verified by direct calculation, using Lemma 4.6. We conclude that the identity also holds for $B=\sigma_{k}^{-1}$, either by another direct calculation, or by using the result for $B=\sigma_{k}$ : since $\phi_{\sigma_{k}}(A)=\Phi_{\sigma_{k}}^{L}(A) \cdot A \cdot \Phi_{\sigma_{k}}^{R}(A)$, we have

$$
A=\Phi_{\sigma_{k}}^{L}\left(\phi_{\sigma_{k}}^{-1}(A)\right) \cdot \phi_{\sigma_{k}}^{-1}(A) \cdot \Phi_{\sigma_{k}}^{R}\left(\phi_{\sigma_{k}}^{-1}(A)\right)=\left(\Phi_{\sigma_{k}^{-1}}^{L}(A)\right)^{-1} \cdot \phi_{\sigma_{k}^{-1}}(A) \cdot\left(\Phi_{\sigma_{k}^{-1}}^{R}(A)\right)^{-1},
$$

where the last equality comes from Corollary 4.5 the desired identity for $B=$ $\sigma_{k}^{-1}$ follows. 
We now show that if the identity holds for $B=B_{1}$ and $B=B_{2}$, then it holds for $B=B_{1} B_{2}$; the proposition then follows by induction. Indeed, assuming the identity for $B=B_{1}$ and $B=B_{2}$, we have

$$
\begin{aligned}
\phi_{B_{1} B_{2}}(A) & =\phi_{B_{1}}\left(\phi_{B_{2}}(A)\right) \\
& =\Phi_{B_{2}}^{L}\left(\phi_{B_{1}}(A)\right) \cdot \phi_{B_{1}}(A) \cdot \Phi_{B_{2}}^{R}\left(\phi_{B_{1}}(A)\right) \\
& =\Phi_{B_{2}}^{L}\left(\phi_{B_{1}}(A)\right) \cdot \Phi_{B_{1}}^{L}(A) \cdot A \cdot \Phi_{B_{1}}^{R}(A) \cdot \Phi_{B_{2}}^{R}\left(\phi_{B_{1}}(A)\right) \\
& =\Phi_{B_{1} B_{2}}^{L}(A) \cdot A \cdot \Phi_{B_{1} B_{2}}^{R}(A),
\end{aligned}
$$

where we use the chain rule for the final equality.

A more geometric proof of Proposition 4.7 is given in [19]. As remarked in Section 2.2. Proposition 4.7 implies that the knot DGA does in fact satisfy $\partial^{2}=0$. It also follows from Proposition 4.7 that the full homomorphism $\phi_{B}$ can be deduced from the matrices $\Phi_{B}^{L}$ and $\Phi_{B}^{R}$, which in turn can be deduced from each other via the conjugation map to be discussed in Section 6.1 Thus we may view $\Phi_{B}^{L}$ and $\Phi_{B}^{R}$ as "square roots" of $\phi_{B}$.

One final result we will need in the future is a reformulation of the knot DGA in terms of an equivalent DGA which has more generators but a slightly simpler definition.

Proposition 4.8 Let $B \in B_{n}$. The knot DGA for $B$ is stable tame isomorphic to the "modified knot DGA" which has generators $\left\{a_{i j} \mid 1 \leq i, j \leq n, i \neq j\right\}$ of degree $0,\left\{b_{i j} \mid 1 \leq i, j \leq n, i \neq j\right\}$ and $\left\{c_{i j}, d_{i j} \mid 1 \leq i, j \leq n\right\}$ of degree 1 , and $\left\{e_{i j}, f_{i j} \mid 1 \leq i, j \leq n\right\}$ of degree 2 , with differential

$$
\begin{aligned}
& \partial A=0 \\
& \partial B=A-\phi_{B}(A) \\
& \partial C=\left(1-\Phi_{B}^{L}(A)\right) \cdot A \\
& \partial D=A \cdot\left(1-\Phi_{B}^{R}(A)\right) \\
& \partial E=B-D-C \cdot \Phi_{B}^{R}(A) \\
& \partial F=B-C-\Phi_{B}^{L}(A) \cdot D .
\end{aligned}
$$

Here we set $b_{i i}=0$ for all $i$.

Proof In the modified knot DGA, replace $e_{i j}$ by $e_{i j}+f_{i j}$ for all $i, j$; after applying the induced tame automorphism, we obtain the same differential, except with $\partial E=C \cdot\left(1-\Phi_{B}^{R}(A)\right)-\left(1-\Phi_{B}^{L}(A)\right) \cdot D$. Now replace $b_{i j}$ by 
$\left(B+C+\Phi_{B}^{L}(A) \cdot D\right)_{i j}$ for $i \neq j$, which constitutes another tame automorphism; the differential is again unchanged, except that $\partial B=0$ (this follows from Proposition 4.7) and $\partial F=B-\operatorname{diag}\left(C+\Phi_{B}^{L}(A) \cdot D\right)$, where diag replaces all non-diagonal entries by 0 . Now we have $\partial f_{i j}=b_{i j}$ for $i \neq j$, and hence, up to stabilization, we may drop the generators $\left\{b_{i j}, f_{i j} \mid i \neq j\right\}$ from the DGA. The resulting DGA is precisely the knot DGA for $B$, after we relabel $c_{i j}, d_{i j}, e_{i j}, f_{i i}$ by $b_{i j}, c_{i j}, d_{i j},-e_{i}$, respectively.

An important consequence of Proposition 4.8 is that the knot DGA for a braid can be obtained from the braid DGA, up to stable tame isomorphism, by adding generators and appropriately extending the definition of the differential.

Corollary 4.9 If $K$ is the closure of $B$, then $H C_{0}(K)$ is a quotient of $H C_{0}(B)$.

Proof Immediate from Propositions 2.4 and 4.8

\subsection{Invariance of $H C_{0}$ for knots}

In this section, we prove the following result.

Theorem 4.10 Up to isomorphism, $H C_{0}(K)$ is a well-defined invariant of a knot $K$.

Proof If $B$ is a braid, we temporarily write $H C_{0}^{\text {knot }}(B)$ for the 0 -dimensional homology of the knot DGA of $B$, as expressed in Proposition 4.2. We wish to show that if $B, \tilde{B}$ are two braids whose closure is the same knot, then $H C_{0}^{\mathrm{knot}}(B), H C_{0}^{\mathrm{knot}}(\tilde{B})$ are isomorphic. By Markov's Theorem, it suffices to check this when $B, \tilde{B}$ are related by one of three operations: conjugation, positive stabilization, and negative stabilization.

Conjugation Here $B, \tilde{B} \in B_{n}$ satisfy $\tilde{B}=C^{-1} B C$ for some $C \in B_{n}$.

Write $H C_{0}^{\text {knot }}(B)=\mathcal{A}_{n} / \mathcal{I}$, where $\mathcal{I}$ is generated by the entries of $\left(1-\Phi_{B}^{L}(A)\right) \cdot A$ and $A \cdot\left(1-\Phi_{B}^{R}(A)\right)$. For clarity, write $H C_{0}^{\mathrm{knot}}(\tilde{B})=\tilde{\mathcal{A}}_{n} / \tilde{\mathcal{I}}$, with $\tilde{\mathcal{A}}_{n}$ generated by $\left\{\tilde{a}_{i j}\right\}$ and $\tilde{\mathcal{I}}$ generated by the entries of $\left(1+\Phi_{\tilde{B}}^{L}(\tilde{A})\right) \cdot \tilde{A}$ and $\tilde{A} \cdot\left(1+\Phi_{\tilde{B}}^{R}(\tilde{A})\right)$, where $\tilde{A}=\left(\tilde{a}_{i j}\right)$ and $\tilde{a}_{i i}=-2$.

We claim that the identification $\tilde{A}=\phi_{C}(A)$, ie, $\tilde{a}_{i j}=\phi_{C}\left(a_{i j}\right)$ for all $i, j$, maps $\tilde{\mathcal{I}}$ into $\mathcal{I}$. This would suffice to prove that $H C_{0}^{\mathrm{knot}}(B) \cong H C_{0}^{\mathrm{knot}}(\tilde{B})$, since 
a symmetrical argument with $B$ and $\tilde{B}$ interchanged shows that the same identification in the other direction also maps $\mathcal{I}$ into $\tilde{\mathcal{I}}$, and hence $\mathcal{I}$ and $\tilde{\mathcal{I}}$ coincide.

Assume that $\tilde{A}=\phi_{C}(A)$. From Proposition 4.4 and Corollary 4.5, we have

$$
\left.\Phi_{\tilde{B}}^{L}(\tilde{A})=\Phi_{B C}^{L}(A) \cdot \Phi_{C^{-1}}^{L}\left(\phi_{C}(A)\right)\right)=\Phi_{C}^{L}\left(\phi_{B}(A)\right) \cdot \Phi_{B}^{L}(A) \cdot\left(\Phi_{C}^{L}(A)\right)^{-1},
$$

and similarly $\Phi_{\tilde{B}}^{R}(\tilde{A})=\left(\Phi_{C}^{R}(A)\right)^{-1} \cdot \Phi_{B}^{R}(A) \cdot \Phi_{C}^{R}\left(\phi_{B}(A)\right)$. It follows by Proposition 4.7 that

$$
\begin{aligned}
\left(1-\Phi_{\tilde{B}}^{L}(\tilde{A})\right) \cdot \tilde{A}= & \left(1-\Phi_{C}^{L}\left(\phi_{B}(A)\right) \cdot \Phi_{B}^{L}(A) \cdot\left(\Phi_{C}^{L}(A)\right)^{-1}\right) \cdot \Phi_{C}^{L}(A) \cdot A \cdot \Phi_{C}^{R}(A) \\
= & \Phi_{C}^{L}(A) \cdot\left(1-\Phi_{B}^{L}(A)\right) \cdot A \cdot \Phi_{C}^{R}(A) \\
& \quad-\left(\Phi_{C}^{L}\left(\phi_{B}(A)\right)-\Phi_{C}^{L}(A)\right) \cdot \Phi_{B}^{L}(A) \cdot A \cdot \Phi_{C}^{R}(A) .
\end{aligned}
$$

Now the entries of $\left(1-\Phi_{B}^{L}(A)\right) \cdot A$ are in $\mathcal{I}$ by definition; on the other hand, the entries of

$\phi_{B}(A)-A=\Phi_{B}^{L}(A) \cdot A \cdot \Phi_{B}^{R}(A)-A=-\left(1-\Phi_{B}^{L}(A)\right) \cdot A \cdot \Phi_{B}^{R}(A)-A \cdot\left(1-\Phi_{B}^{R}(A)\right)$ are in $\mathcal{I}$, and thus so are the entries of $\Phi_{C}^{L}\left(\phi_{B}(A)\right)-\Phi_{C}^{L}(A)$. Hence the entries of $\left(1-\Phi_{\tilde{B}}^{L}(\tilde{A})\right) \cdot \tilde{A}$ are in $\mathcal{I}$. Similarly, the entries of $\tilde{A} \cdot\left(1-\Phi_{\tilde{B}}^{R}(\tilde{A})\right)$ are also in $\mathcal{I}$, and therefore $\tilde{\mathcal{I}} \subset \mathcal{I}$, as desired.

Positive stabilization Here $\tilde{B}$ is obtained from $B$ by adding an extra strand, which we label 0 , to the braid, and setting $\tilde{B}=B \sigma_{0}$.

Analogously to before, write $H C_{0}^{\text {knot }}(B)=\mathcal{A}_{n} / \mathcal{I}$ and $H C_{0}^{\text {knot }}(\tilde{B})=\tilde{\mathcal{A}} / \tilde{\mathcal{I}}$, where now $\tilde{\mathcal{A}}$ is obtained from $\mathcal{A}_{n}$ by adding generators $a_{i 0}, a_{0 i}$ for $1 \leq$ $i \leq n$. Abbreviate $\Phi_{B}^{L}(A), \Phi_{B}^{R}(A)$ by $\Phi^{L}, \Phi^{R}$. By using Proposition 4.4 and Lemma 4.6, we can easily compute the matrices for $\Phi_{B \sigma_{0}}^{L}, \Phi_{B \sigma_{0}}^{R}$ in terms of $\Phi^{L}, \Phi^{R}$ :

$$
\begin{array}{lll}
\left(\Phi_{B \sigma_{0}}^{L}\right)_{00}=-\phi_{B}\left(a_{10}\right) & \left(\Phi_{B \sigma_{0}}^{R}\right)_{00}=-\phi_{B}\left(a_{01}\right) \\
\left(\Phi_{B \sigma_{0}}^{L}\right)_{0 i}=-\Phi_{1 i}^{L} & \left(\Phi_{B \sigma_{0}}^{R}\right)_{i 0}=-\Phi_{i 1}^{R} \\
\left(\Phi_{B \sigma_{0}}^{L}\right)_{10}=1 & \left(\Phi_{B \sigma_{0}}^{R}\right)_{01}=1 \\
\left(\Phi_{B \sigma_{0}}^{L}\right)_{1 i}=0 & \left(\Phi_{B \sigma_{0}}^{R}\right)_{i 1}=0 \\
\left(\Phi_{B \sigma_{0}}^{L}\right)_{j 0}=0 & \left(\Phi_{B \sigma_{0}}^{R}\right)_{0 j}=0 \\
\left(\Phi_{B \sigma_{0}}^{L}\right)_{j i}=\Phi_{j i}^{L} & \left(\Phi_{B \sigma_{0}}^{R}\right)_{i j}=\Phi_{i j}^{R} .
\end{array}
$$

Here $i, j$ are any indices such that $i \geq 1$ and $j \geq 2$. It is then straightforward to calculate the generators of $\tilde{\mathcal{I}}$; they are the entries of $\left(1-\Phi_{\tilde{B}}^{L}(A)\right) \cdot A$ and 
$A \cdot\left(1-\Phi_{\tilde{B}}^{R}(A)\right)$, which we write as $\tilde{\partial} \tilde{B}$ and $\tilde{\partial} \tilde{C}$ for future use in Section 5.2 .

$$
\begin{array}{rlrl}
\tilde{\partial} \tilde{b}_{00} & =-2-\phi_{B}\left(a_{10}\right) & & \tilde{\partial} \tilde{c}_{00}=-2-\phi_{B}\left(a_{01}\right) \\
\tilde{\partial} \tilde{b}_{0 i} & =a_{0 i}+\phi_{B}\left(a_{10}\right) a_{0 i}+\Phi_{1 \ell}^{L} a_{\ell i} & & \tilde{\partial} \tilde{c}_{i 0}=a_{i 0}+a_{i 0} \phi_{B}\left(a_{01}\right)+a_{i \ell} \Phi_{\ell 1}^{R} \\
\tilde{\partial} \tilde{b}_{10} & =a_{10}+2 & \tilde{\partial} \tilde{c}_{01}=a_{01}+2 \\
\tilde{\partial} \tilde{b}_{1 i} & =a_{1 i}-a_{0 i} & & \tilde{\partial} \tilde{c}_{i 1}=a_{i 1}-a_{i 0} \\
\tilde{\partial} \tilde{b}_{j 0} & =a_{j 0}-\Phi_{j \ell}^{L} a_{\ell 0} & & \tilde{\partial} \tilde{c}_{0 j}=a_{0 j}-a_{0 \ell} \Phi_{\ell j}^{R} \\
\tilde{\partial} \tilde{b}_{j i} & =a_{j i}-\Phi_{j \ell}^{L} a_{\ell i} & & \tilde{\partial} \tilde{c}_{i j}=a_{i j}-a_{i \ell} \Phi_{\ell j}^{R},
\end{array}
$$

where, as before, $i \geq 1$ and $j \geq 2$, and any monomial involving the index $\ell$ is understood to be summed from $\ell=1$ to $\ell=n$. (To obtain the expressions for $\tilde{b}_{00}$ and $\tilde{c}_{00}$, we use the facts that $\phi_{B}\left(a_{10}\right)=\Phi_{1 \ell}^{L} a_{\ell 0}$ and $\phi_{B}\left(a_{01}\right)=a_{0 \ell} \Phi_{\ell 1}^{R}$, which follow from the definitions of $\Phi^{L}$ and $\Phi^{R}$.)

In $\tilde{\mathcal{A}} / \tilde{\mathcal{I}}$, we thus have $a_{0 i}=a_{1 i}$ and $a_{i 0}=a_{i 1}$ for $i \geq 1$; in particular, $a_{01}=$ $a_{10}=-2$. Using these relations to replace all generators of the form $a_{0 i}$ and $a_{i 0}$, we find that the remaining relations in $\tilde{\mathcal{A}} / \tilde{\mathcal{I}}$ give precisely the generators of $\mathcal{I}$, namely $a_{i j}-\Phi_{i \ell}^{L} a_{\ell j}$ and $a_{i j}-a_{i \ell} \Phi_{\ell j}^{R}$ for $1 \leq i, j \leq n$. Thus $\mathcal{A}_{n} / \mathcal{I} \cong \tilde{\mathcal{A}} / \tilde{\mathcal{I}}$, as desired.

Negative stabilization Here $\tilde{B}$ is obtained from $B$ by adding an extra strand, which we label 0 , to the braid, and setting $\tilde{B}=B \sigma_{0}^{-1}$.

This case is very similar to the case of positive stabilization, with a slightly different computation. Use the same notation as for positive stabilization; then we have

$$
\begin{array}{ll}
\left(\Phi_{B \sigma_{0}}^{L}\right)_{00}=0 & \left(\Phi_{B \sigma_{0}}^{R}\right)_{00}=0 \\
\left(\Phi_{B \sigma_{0}}^{L}\right)_{0 i}=\Phi_{1 i}^{L} & \left(\Phi_{B \sigma_{0}}^{R}\right)_{i 0}=\Phi_{i 1}^{R} \\
\left(\Phi_{B \sigma_{0}}^{L}\right)_{10}=-1 & \left(\Phi_{B \sigma_{0}}^{R}\right)_{01}=-1 \\
\left(\Phi_{B \sigma_{0}}^{L}\right)_{1 i}=-\phi_{B}\left(a_{01}\right) \Phi_{1 i}^{L} & \left(\Phi_{B \sigma_{0}}^{R}\right)_{i 1}=-\Phi_{i 1}^{R} \phi_{B}\left(a_{10}\right) \\
\left(\Phi_{B \sigma_{0}}^{L}\right)_{j 0}=0 & \left(\Phi_{B \sigma_{0}}^{R}\right)_{0 j}=0 \\
\left(\Phi_{B \sigma_{0}}^{L}\right)_{j i}=\Phi_{j i}^{L} & \left(\Phi_{B \sigma_{0}}^{R}\right)_{i j}=\Phi_{i j}^{R}
\end{array}
$$

for $i \geq 1$ and $j \geq 2$, and so the generators of $\tilde{\mathcal{I}}$ are

$$
\begin{aligned}
\tilde{\partial} \tilde{b}_{00} & =-2-\phi_{B}\left(a_{10}\right) & & \tilde{\partial} \tilde{c}_{00}=-2-\phi_{B}\left(a_{01}\right) \\
\tilde{\partial} \tilde{b}_{0 i} & =a_{0 i}-\Phi_{1 \ell}^{L} a_{\ell i} & & \tilde{\partial} \tilde{c}_{i 0}=a_{i 0}-a_{i \ell} \Phi_{\ell 1}^{R} \\
\tilde{\partial} \tilde{b}_{10} & =a_{10}-2+\phi_{B}\left(a_{01}\right) \phi_{B}\left(a_{10}\right) & & \tilde{\partial} \tilde{c}_{01}=a_{01}-2+\phi_{B}\left(a_{01}\right) \phi_{B}\left(a_{10}\right)
\end{aligned}
$$




$$
\begin{aligned}
\tilde{\partial} \tilde{b}_{1 i} & =a_{1 i}+a_{0 i}+\phi_{B}\left(a_{01}\right) \Phi_{1 \ell}^{L} a_{\ell i} & & \tilde{\partial} \tilde{c}_{i 1}=a_{i 1}+a_{i 0}+a_{i \ell} \Phi_{\ell 1}^{R} \phi_{B}\left(a_{10}\right) \\
\tilde{\partial} \tilde{b}_{j 0} & =a_{j 0}-\Phi_{j \ell}^{L} a_{\ell 0} & & \tilde{\partial} \tilde{c}_{0 j}=a_{0 j}-a_{0 \ell} \Phi_{\ell j}^{R} \\
\tilde{\partial} \tilde{b}_{j i} & =a_{j i}-\Phi_{j \ell}^{L} a_{\ell i} & & \tilde{\partial} \tilde{c}_{i j}=a_{i j}-a_{i \ell} \Phi_{\ell j}^{R} .
\end{aligned}
$$

In $\tilde{\mathcal{A}} / \tilde{\mathcal{I}}$, we have $a_{0 i}=\Phi_{1 \ell}^{L} a_{\ell i}$ and $a_{i 0}=a_{i \ell} \Phi_{\ell 1}^{R}$. When we use these relations to eliminate $a_{0 i}, a_{i 0}$, we find that the remaining relations in $\tilde{\mathcal{A}} / \tilde{\mathcal{I}}$ are precisely the relations in $\mathcal{A}_{n} / \mathcal{I}$, as before. Hence $\tilde{\mathcal{A}} / \tilde{\mathcal{I}} \cong \mathcal{A}_{n} / \mathcal{I}$.

We have shown that $H C_{0}^{\mathrm{knot}}(B)$ is invariant under the Markov moves, and hence it gives a well-defined knot invariant, as desired.

\section{Invariance proofs for braid and knot DGAs}

In this section, we prove Theorems 2.7 and 2.10, the invariance results for braid and knot DGAs. As mentioned before, the proofs are essentially more involved versions of the corresponding proofs for $H C_{0}$ given in Section 4

\subsection{Proof of Theorem 2.7}

It suffices to establish Theorem 2.7 under the assumption that $\tilde{B}=\sigma_{k}^{-1} B \sigma_{k}$ for some $k$. Then the braid DGAs for $B$ and $\tilde{B}$ are generated by $a_{i j}, b_{i j}$ and $\tilde{a}_{i j}, \tilde{b}_{i j}$, respectively, for $1 \leq i \neq j \leq n$. If we abbreviate $\phi_{B}$ by $\phi$ and $\phi_{\sigma_{k}}$ by $\phi_{k}$, then the differentials for the braid DGAs are given by $\partial b_{i j}=a_{i j}-\phi\left(a_{i j}\right)$ and $\tilde{\partial} \tilde{b}_{i j}=\tilde{a}_{i j}-\phi_{k}^{-1} \phi \phi_{k}\left(\tilde{a}_{i j}\right)$. (By abusing notation, we will consider $\phi$ and $\phi_{k}$ to act either on the algebra generated by the $a_{i j}$ or on the algebra generated by the $\tilde{a}_{i j}$.) We will find a tame isomorphism between the algebras which sends one differential to the other.

Set $\tilde{a}_{i j}=\phi_{k}\left(a_{i j}\right)$ for all $i, j$. Then

$$
\tilde{\partial} \tilde{b}_{i j}=\tilde{a}_{i j}-\phi_{k}^{-1} \phi \phi_{k}\left(\tilde{a}_{i j}\right)=\phi_{k}\left(a_{i j}\right)-\phi \phi_{k}\left(a_{i j}\right) .
$$

Now make the following identifications:

$$
\begin{aligned}
\tilde{b}_{k i} & =-b_{k+1, i}-\phi\left(a_{k+1, k}\right) b_{k i}-b_{k+1, k} a_{k i} & & i \neq k, k+1 \\
\tilde{b}_{i k} & =-b_{i, k+1}-b_{i k} \phi\left(a_{k, k+1}\right)-a_{i k} b_{k, k+1} & & i \neq k, k+1 \\
\tilde{b}_{k+1, i} & =b_{k i} & & i \neq k, k+1 \\
\tilde{b}_{i, k+1} & =b_{i k} & & i \neq k, k+1 \\
\tilde{b}_{k, k+1} & =b_{k+1, k} & &
\end{aligned}
$$




$$
\begin{array}{rlrl}
\tilde{b}_{k+1, k} & =b_{k, k+1} \\
\tilde{b}_{i j} & =b_{i j} & i, j \neq k, k+1 .
\end{array}
$$

It is straightforward to check from $\partial b_{i j}=a_{i j}-\phi\left(a_{i j}\right)$ that $\partial \tilde{b}_{i j}=\phi_{k}\left(a_{i j}\right)-$ $\phi \phi_{k}\left(a_{i j}\right)$ for all $i, j$. For instance, for $i \neq k, k+1$, we have

$$
\begin{aligned}
\partial \tilde{b}_{i k} & =-\partial b_{i, k+1}-\left(\partial b_{i k}\right) \phi\left(a_{k, k+1}\right)-a_{i k}\left(\partial b_{k, k+1}\right) \\
& =-a_{i, k+1}+\phi\left(a_{i, k+1}\right)+\phi\left(a_{i k}\right) \phi\left(a_{k, k+1}\right)-a_{i k} a_{k, k+1} \\
& =\phi_{k}\left(a_{i k}\right)-\phi \phi_{k}\left(a_{i k}\right) .
\end{aligned}
$$

Using the above definitions for $\tilde{a}_{i j}, \tilde{b}_{i j}$ in terms of $a_{i j}, b_{i j}$, we conclude that $\partial=\tilde{\partial}$, and so the map sending $a_{i j}$ to $\tilde{a}_{i j}$ and $b_{i j}$ to $\tilde{b}_{i j}$ sends $\partial$ to $\tilde{\partial}$. On the other hand, it is easy to check that this map is tame; the map on the $a_{i j}$ is given by the tame isomorphism $\phi_{k}$, while the map on the $b_{i j}$ is tame by inspection of the above definition for $\tilde{b}_{i j}$ in terms of $b_{i j}$. Thus the braid DGAs for $B$ and $\sigma_{k}^{-1} B \sigma_{k}$ are tamely isomorphic, as desired.

\subsection{Proof of Theorem 2.10}

As in the proof of Theorem 4.10, it suffices to show that the equivalence class of the knot DGA of a braid is invariant under the Markov moves.

Conjugation Let $B \in B_{n}$ be a braid, and let $\tilde{B}=\sigma_{k}^{-1} B \sigma_{k}$; we show that the modified knot DGAs (see Proposition 4.8) for $B$ and $\tilde{B}$ are tamely isomorphic.

For clarity, we distinguish between the modified knot DGAs for $B$ and $\tilde{B}$ by using tildes on the generators and differential of the knot DGA for $\tilde{B}$. As in the proof of Theorem 2.7, we will exhibit an identification between the two sets of generators so that $\partial=\tilde{\partial}$.

By Lemma 4.6] the matrices $\Phi_{\sigma_{k}}^{L}(A)-\Phi_{\sigma_{k}}^{L}\left(\phi_{B}(A)\right)$ and $\Phi_{\sigma_{k}}^{R}(A)-\Phi_{\sigma_{k}}^{R}\left(\phi_{B}(A)\right)$ are both identically zero except in the $(k, k)$ entry, where they are $-a_{k+1, k}+$ $\phi_{B}\left(a_{k+1, k}\right)$ and $-a_{k, k+1}+\phi_{B}\left(a_{k, k+1}\right)$, respectively. Hence if we define $n \times n$ matrices $\Theta_{k}^{L}(A)$ and $\Theta_{k}^{R}(A)$ to be zero except in the $(k, k)$ entry, where they are $-b_{k+1, k}$ and $-b_{k, k+1}$, respectively, then we have

$$
\partial \Theta_{k}^{L}(A)=\Phi_{\sigma_{k}}^{L}(A)-\Phi_{\sigma_{k}}^{L}\left(\phi_{B}(A)\right) \quad \text { and } \quad \partial \Theta_{k}^{R}(A)=\Phi_{\sigma_{k}}^{R}(A)-\Phi_{\sigma_{k}}^{R}\left(\phi_{B}(A)\right) .
$$

Identify the $\tilde{a}_{i j}, \tilde{b}_{i j}$ with the $a_{i j}, b_{i j}$ using the same map as in the proof of Theorem 2.7 in Section 5.1] then $\partial$ and $\tilde{\partial}$ agree on $\tilde{a}_{i j}$ and $\tilde{b}_{i j}$. Now set

$$
\tilde{C}=\Phi_{\sigma_{k}}^{L}\left(\phi_{B}(A)\right) \cdot C \cdot \Phi_{\sigma_{k}}^{R}(A)+\Theta_{k}^{L}(A) \cdot A \cdot \Phi_{\sigma_{k}}^{R}(A),
$$




$$
\begin{aligned}
& \tilde{D}=\Phi_{\sigma_{k}}^{L}(A) \cdot D \cdot \Phi_{\sigma_{k}}^{R}\left(\phi_{B}(A)\right)+\Phi_{\sigma_{k}}^{L}(A) \cdot A \cdot \Theta_{k}^{R}(A), \\
& \tilde{E}=\Phi_{\sigma_{k}}^{L}\left(\phi_{B}(A)\right) \cdot E \cdot \Phi_{\sigma_{k}}^{R}\left(\phi_{B}(A)\right)-\Theta_{k}^{L}(A) \cdot D \cdot \Phi_{\sigma_{k}}^{R}\left(\phi_{B}(A)\right) \quad+\Theta_{k}^{L}(A) \cdot \Theta_{k}^{R}(A), \\
& \tilde{F}=\Phi_{\sigma_{k}}^{L}\left(\phi_{B}(A)\right) \cdot F \cdot \Phi_{\sigma_{k}}^{R}\left(\phi_{B}(A)\right)+\Phi_{\sigma_{k}}^{L}\left(\phi_{B}(A)\right) \cdot C \cdot \Theta_{k}^{R}(A) \\
& \quad+\Theta_{k}^{L}(A) \cdot(1+A) \cdot \Theta_{k}^{R}(A) .
\end{aligned}
$$

We claim that these identifications make $\partial$ and $\tilde{\partial}$ agree on $\tilde{c}_{i j}, \tilde{d}_{i j}, \tilde{e}_{i j}, \tilde{f}_{i j}$, which implies that $\partial=\tilde{\partial}$ on the entire algebra. We will check that $\tilde{\partial} \tilde{C}=\partial \tilde{C}$ and $\tilde{\partial} \tilde{E}=\partial \tilde{E} ;$ the proofs for $D$ and $F$ are completely analogous.

By Proposition 4.4 and Corollary 4.5. we have

$$
\begin{aligned}
\Phi_{\tilde{B}}^{L}(\tilde{A}) & =\Phi_{\sigma_{k}^{-1} B \sigma_{k}}^{L}\left(\phi_{\sigma_{k}}(A)\right)=\Phi_{B \sigma_{k}}^{L}(A) \cdot \Phi_{\sigma_{k}^{-1}}^{L}\left(\phi_{\sigma_{k}}(A)\right) \\
& =\Phi_{\sigma_{k}}^{L}\left(\phi_{B}(A)\right) \cdot \Phi_{B}^{L}(A) \cdot\left(\Phi_{\sigma_{k}}^{L}(A)\right)^{-1},
\end{aligned}
$$

and similarly

$$
\Phi_{\tilde{B}}^{R}(\tilde{A})=\left(\Phi_{\sigma_{k}}^{R}(A)\right)^{-1} \cdot \Phi_{B}^{R}(A) \cdot \Phi_{\sigma_{k}}^{R}\left(\phi_{B}(A)\right) .
$$

Recall from Proposition 4.7 that $\tilde{A}=\phi_{\sigma_{k}}(A)=\Phi_{\sigma_{k}}^{L}(A) \cdot A \cdot \Phi_{\sigma_{k}}^{R}(A)$; it follows that

$$
\begin{aligned}
\partial \tilde{C}= & \Phi_{\sigma_{k}}^{L}\left(\phi_{B}(A)\right) \cdot\left(1-\Phi_{B}^{L}(A)\right) \cdot A \cdot \Phi_{\sigma_{k}}^{R}(A) \\
& \quad+\left(\Phi_{\sigma_{k}}^{L}(A)-\Phi_{\sigma_{k}}^{L}\left(\phi_{B}(A)\right)\right) \cdot A \cdot \Phi_{\sigma_{k}}^{R}(A) \\
= & \left(\Phi_{\sigma_{k}}^{L}(A)-\Phi_{\sigma_{k}}^{L}\left(\phi_{B}(A)\right) \cdot \Phi_{B}^{L}(A)\right) \cdot A \cdot \Phi_{\sigma_{k}}^{R}(A) \\
= & \left(1-\Phi_{\tilde{B}}^{L}(\tilde{A})\right) \cdot \tilde{A} \\
= & \tilde{\partial} \tilde{C}
\end{aligned}
$$

and

$$
\begin{aligned}
\partial \tilde{E}= & \Phi_{\sigma_{k}}^{L}\left(\phi_{B}(A)\right) \cdot\left(B-D-C \cdot \Phi_{B}^{R}(A)\right) \cdot \Phi_{\sigma_{k}}^{R}\left(\phi_{B}(A)\right) \\
& \quad-\left(\Phi_{\sigma_{k}}^{L}(A)-\Phi_{\sigma_{k}}^{L}\left(\phi_{B}(A)\right)\right) \cdot D \cdot \Phi_{\sigma_{k}}^{R}\left(\phi_{B}(A)\right) \\
& \quad+\Theta_{k}^{L}(A) \cdot A \cdot\left(1-\Phi_{B}^{R}(A)\right) \cdot \Phi_{\sigma_{k}}^{R}\left(\phi_{B}(A)\right)+\partial\left(\Theta_{k}^{L}(A) \cdot \Theta_{k}^{R}(A)\right) \\
& =\tilde{B}-\tilde{D}-\tilde{C} \cdot \Phi_{\tilde{B}}^{R}(\tilde{A}) \\
= & \tilde{\partial} \tilde{E},
\end{aligned}
$$

where we use the fact (a direct computation from the definition of $\tilde{b}_{i j}$ ) that

$$
\begin{array}{r}
\tilde{B}=\Phi_{\sigma_{k}}^{L}\left(\phi_{B}(A)\right) \cdot B \cdot \Phi_{\sigma_{k}}^{R}\left(\phi_{B}(A)\right)+\Phi_{\sigma_{k}}^{L}(A) \cdot A \cdot \Theta_{k}^{R}(A) \\
+\Theta_{k}^{L}(A) \cdot A \cdot \Phi_{\sigma_{k}}^{R}\left(\phi_{B}(A)\right)+\partial\left(\Theta_{k}^{L}(A) \cdot \Theta_{k}^{R}(A)\right) .
\end{array}
$$


Now that we have established that $\partial=\tilde{\partial}$ on the entire algebra, it only remains to verify that the identification of variables $A \mapsto \tilde{A}$, etc., constitutes a tame isomorphism. This is straightforward; as in the proof of Theorem 2.7. the map $A \mapsto \tilde{A}, B \mapsto \tilde{B}$ is a tame automorphism. We can then send $C$ to $\tilde{C}$ via the composition of tame automorphisms

$$
\begin{aligned}
& C \mapsto C+\Theta_{k}^{L}(A) \cdot A \cdot \Phi_{\sigma_{k}}^{R}(A), \\
& C \mapsto \Phi_{\sigma_{k}}^{L}\left(\phi_{B}(A)\right) \cdot C, \\
& C \mapsto C \cdot \Phi_{\sigma_{k}}^{R}(A),
\end{aligned}
$$

of which the last two are tame because of Lemma 4.6, and similarly for $D \mapsto \tilde{D}$. Finally, we can send $E$ to $\tilde{E}$ via the composition of tame automorphisms

$$
\begin{aligned}
& E \mapsto E-\Theta_{k}^{L}(A) \cdot D \cdot \Phi_{\sigma_{k}}^{R}\left(\phi_{B}(A)\right)+\Theta_{k}^{L}(A) \cdot \Theta_{k}^{R}(A), \\
& E \mapsto \Phi_{\sigma_{k}}^{L}\left(\phi_{B}(A)\right) \cdot E, \\
& E \mapsto E \cdot \Phi_{\sigma_{k}}^{R}\left(\phi_{B}(A)\right),
\end{aligned}
$$

and similarly for $F \mapsto \tilde{F}$.

Positive stabilization Let $B \in B_{n}$ be a braid; as in the proof of Theorem 4.10 we denote stabilization by adding a strand labelled 0 and considering the braid $\tilde{B}=B \sigma_{0}$. We will prove that the knot DGAs for $B$ and $\tilde{B}$ are stable tame isomorphic.

Denote the knot DGA for $B$ by $(\mathcal{A}, \partial)$ and the knot DGA for $\tilde{B}$ by $(\tilde{\mathcal{A}}, \tilde{\partial})$, where the generators of $\tilde{\mathcal{A}}$ have tildes for notational clarity. Thus $\tilde{\mathcal{A}}$ is generated by $\left\{\tilde{a}_{i j} \mid 0 \leq i \neq j \leq n\right\}$ in degree 0 , and so forth. Note that $\tilde{\mathcal{A}}$ has more generators than $\mathcal{A}$ does; we will establish that we can obtain $(\tilde{\mathcal{A}}, \tilde{\partial})$ from $(\mathcal{A}, \partial)$ by a suitable number of stabilizations.

We will progressively identify generators of $\mathcal{A}$ and $\tilde{\mathcal{A}}$ so that $\partial$ and $\tilde{\partial}$ agree. The first identification is to drop all tildes on the $\tilde{a}_{i j}$ generators; that is, set $a_{i j}=\tilde{a}_{i j}$ for all $i, j \geq 0, i \neq j$. Note that this introduces $2 n$ generators $a_{0 j}$ and $a_{i 0}$ not in $\mathcal{A}$.

The matrices $\Phi_{B \sigma_{0}}^{L}$ and $\Phi_{B \sigma_{0}}^{R}$ were computed in the proof of Theorem 4.10 We can then compute $\tilde{\partial}$ in terms of $\Phi^{L}=\Phi_{B}^{L}$ and $\Phi^{R}=\Phi_{B}^{R}$. For $\tilde{b}_{i j}$ and $\tilde{c}_{i j}$, we already calculated $\tilde{\partial}$ in the proof of Theorem 4.10. For completeness, we include here the differentials of $\tilde{d}_{i j}$ and $\tilde{e}_{i j}$ :

$$
\begin{aligned}
& \tilde{\partial} \tilde{d}_{00}=\tilde{b}_{00}-\tilde{c}_{00}+\tilde{b}_{00} \phi_{B}\left(a_{01}\right)+\tilde{b}_{0 \ell} \Phi_{\ell 1}^{R}-\phi_{B}\left(a_{10}\right) \tilde{c}_{00}-\Phi_{1 \ell}^{L} \tilde{c}_{\ell 0} \\
& \tilde{\partial} \tilde{d}_{01}=\tilde{b}_{01}-\tilde{c}_{01}-\tilde{b}_{00}-\phi_{B}\left(a_{10}\right) \tilde{c}_{01}-\Phi_{1 \ell}^{L} \tilde{c}_{\ell 1}
\end{aligned}
$$




$$
\begin{aligned}
\tilde{\partial} \tilde{d}_{0 j} & =\tilde{b}_{0 j}-\tilde{c}_{0 j}-\tilde{b}_{0 \ell} \Phi_{\ell j}^{R}-\phi_{B}\left(a_{10}\right) \tilde{c}_{0 j}-\Phi_{1 \ell}^{L} \tilde{c}_{\ell j} \\
\tilde{\partial} \tilde{d}_{10} & =\tilde{b}_{10}-\tilde{c}_{10}+\tilde{c}_{00}+\tilde{b}_{10} \phi_{B}\left(a_{01}\right)+\tilde{b}_{1 \ell} \Phi_{\ell 1}^{R} \\
\tilde{\partial} \tilde{d}_{11} & =\tilde{b}_{11}-\tilde{c}_{11}-\tilde{b}_{10}+\tilde{c}_{01} \\
\tilde{\partial} \tilde{d}_{1 j} & =\tilde{b}_{1 j}-\tilde{c}_{1 j}+\tilde{c}_{0 j}-\tilde{b}_{1 \ell} \Phi_{\ell 1}^{R} \\
\tilde{\partial} \tilde{d}_{j 0} & =\tilde{b}_{j 0}-\tilde{c}_{j 0}+\tilde{b}_{j 0} \phi_{B}\left(a_{01}\right)+\tilde{b}_{j \ell} \Phi_{\ell 1}^{R}+\Phi_{j \ell}^{L} \tilde{\ell}_{\ell 0} \\
\tilde{\partial} \tilde{d}_{j 1} & =\tilde{b}_{j 1}-\tilde{c}_{j 1}-\tilde{b}_{j 0}+\Phi_{j \ell}^{L} \tilde{c}_{\ell 1} \\
\tilde{\partial} \tilde{d}_{j_{1} j_{2}} & =\tilde{b}_{j_{1} j_{2}}-\tilde{c}_{j_{1} j_{2}}-\tilde{b}_{j_{1} \ell} \Phi_{\ell j_{2}}^{R}+\Phi_{j_{1} \ell}^{L} \tilde{c}_{\ell j_{2}} \\
\tilde{\partial} \tilde{e}_{0} & =\tilde{b}_{00}-\phi_{B}\left(a_{10}\right) \tilde{c}_{00}-\Phi_{1 \ell}^{L} \tilde{c}_{\ell 0} \\
\tilde{\partial} \tilde{e}_{1} & =\tilde{b}_{11}+\tilde{c}_{01} \\
\tilde{\partial} \tilde{e}_{j} & =\tilde{b}_{j j}+\Phi_{j \ell}^{L} \tilde{c}_{\ell j},
\end{aligned}
$$

where $j, j_{1}, j_{2} \geq 2$ and, as usual, all monomials involving $\ell$ are summed from $\ell=1$ to $\ell=n$.

If we set

$$
\begin{array}{ll}
b_{1 i}=-\tilde{b}_{0 i}+\tilde{b}_{1 i}-\tilde{b}_{00} a_{0 i} & c_{i 1}=-\tilde{c}_{i 0}+\tilde{c}_{i 1}-a_{i 0} \tilde{c}_{00} \\
b_{j i}=\tilde{b}_{j i} & c_{i j}=\tilde{c}_{i j}
\end{array}
$$

for $i \geq 1$ and $j \geq 2$, then it is easy to check, from the expressions for $\tilde{\partial} \tilde{b}_{i j}$, $\tilde{\partial} \tilde{c}_{i j}$ given in the proof of Theorem 4.10, that $\tilde{\partial} b_{i j}=\partial b_{i j}$ and $\tilde{\partial} c_{i j}=\partial c_{i j}$ for all $i, j \geq 1$. Next set

$$
\begin{aligned}
d_{11} & =\tilde{d}_{00}-\tilde{d}_{01}+\tilde{d}_{11}-\tilde{d}_{10}+\tilde{b}_{00} \tilde{c}_{01}+\tilde{b}_{10} \tilde{c}_{00} \\
d_{1 j} & =-\tilde{d}_{0 j}+\tilde{d}_{1 j}+\tilde{b}_{00} \tilde{c}_{0 j} \\
d_{j 1} & =-\tilde{d}_{j 0}+\tilde{d}_{j 1}+\tilde{b}_{j 0} \tilde{c}_{00} \\
d_{j_{1} j_{2}} & =\tilde{d}_{j_{1} j_{2}} \\
e_{1} & =\tilde{e}_{0}+\tilde{e}_{1}-\tilde{d}_{01}+\tilde{b}_{00} \tilde{c}_{01} \\
e_{j} & =\tilde{e}_{j} \\
d_{00} & =\tilde{d}_{10}-\tilde{d}_{11}+\tilde{d}_{01}-\tilde{b}_{10} \tilde{c}_{00}-\tilde{b}_{00} \tilde{c}_{01} \\
e_{0} & =\tilde{d}_{01}-\tilde{e}_{1}-\tilde{b}_{00} \tilde{c}_{01} \\
d_{10}=\tilde{d}_{11} & -\tilde{e}_{1} \quad d_{01}=\tilde{e}_{1} \quad d_{0 j}=\tilde{d}_{1 j} \quad d_{j 0}=\tilde{d}_{j 1}
\end{aligned}
$$

for $j, j_{1}, j_{2} \geq 2$, and then successively set

$$
\begin{aligned}
& b_{10}=\tilde{b}_{10}+\tilde{c}_{11} \\
& \text { and } \quad c_{01}=\tilde{c}_{01}+\tilde{b}_{11} ; \\
& b_{00}=\tilde{b}_{00}-b_{11}-\Phi_{1 \ell}^{L} \tilde{c}_{\ell 1} \\
& \text { and } \quad c_{00}=\tilde{c}_{00}-c_{11}-\tilde{b}_{1 \ell} \Phi_{\ell 1}^{R} \text {; }
\end{aligned}
$$




$$
\begin{aligned}
& b_{j 0}=\tilde{b}_{j 0}+\tilde{c}_{j 1}-b_{j 1}-\Phi_{j \ell}^{L} \tilde{c}_{\ell 1} \quad \text { and } \quad c_{0 j}=\tilde{c}_{0 j}+\tilde{b}_{1 j}-c_{1 j}-\tilde{b}_{1 \ell} \Phi_{\ell j}^{R} ; \\
& b_{0 i}=\tilde{b}_{1 i} \quad \text { and } \quad c_{i 0}=\tilde{c}_{i 1}
\end{aligned}
$$

for $i \geq 1$ and $j \geq 2$.

Under the above identifications, the map sending the tilde variables to the corresponding non-tilde variables is a tame automorphism on $\tilde{\mathcal{A}}$. To see this, start with the generators of $\tilde{\mathcal{A}}$, and now perform the following changes of variables. First introduce new variables $b_{1 i}, c_{i 1}$ for $i \geq 1$, and then eliminate the generators $\tilde{b}_{0 i}, \tilde{c}_{i 0}$ by using the first identifications: $\tilde{b}_{0 i}=-b_{1 i}+\tilde{b}_{1 i}-\tilde{b}_{00} a_{0 i}$ and $\tilde{c}_{i 0}=-c_{i 1}+\tilde{c}_{i 1}-a_{i 0} \tilde{c}_{00}$. Now successively continue this process using the given identifications, from first to last; for each identification, introduce a new generator given by the left hand side, and use the relation to eliminate the generator given by the first term on the right hand side. This can be done because no eliminated generator appears in successive identifications. The final result is that all tilde variables are eliminated and replaced by non-tilde variables. It follows that the map sending tilde generators to non-tilde generators is tame.

On the other hand, a tedious but straightforward series of calculations demonstrates that $\partial=\tilde{\partial}$ after the above identifications have been made, where we set $\partial b_{0 i}=-a_{0 i}+a_{1 i}, \partial c_{i 0}=-a_{i 0}+a_{i 1}, \partial e_{0}=b_{00}, \partial d_{00}=b_{00}-c_{00}, \partial d_{i 0}=-b_{i 0}$, $\partial d_{0 i}=c_{0 i}$ for $i \geq 1$. It follows that $(\tilde{\mathcal{A}}, \tilde{\partial})$ is tamely isomorphic to the result of stabilizing $(\mathcal{A}, \partial)$ by appending generators $a_{0 i}, a_{i 0}, b_{00}, b_{0 i}, b_{i 0}, c_{00}, c_{0 i}$, $c_{i 0}, d_{00}, d_{0 i}, d_{i 0}, e_{0}$, and extending $\partial$ as in the previous sentence. (This corresponds to $2 n$ stabilizations of degree 0 and $2 n+2$ stabilizations of degree 1.) This completes the proof that the knot DGAs for $B$ and $\tilde{B}$ are stable tame isomorphic.

Negative stabilization We now wish to prove that the knot DGAs for $B$ and $\tilde{B}=B \sigma_{0}^{-1}$ are stable tame isomorphic; this proof is entirely similar to the proof for positive stabilization. We omit the calculations, but the sequence of successive identifications is as follows, where indices satisfy $i \geq 1$ and $j, j_{1}, j_{2} \geq$ 2 , and all monomials involving $\ell$ are understood to be summed over $\ell \geq 1$ :

$$
\begin{aligned}
b_{1 i} & =\tilde{b}_{1 i}-\tilde{b}_{0 i}+\tilde{c}_{00} \Phi_{1 \ell}^{L} a_{\ell i} & c_{i 1} & =\tilde{c}_{i 1}-\tilde{c}_{i 0}+a_{i \ell} \Phi_{\ell 1}^{R} \tilde{b}_{00} \\
b_{j i} & =\tilde{b}_{j i} & c_{i j} & =\tilde{c}_{i j},
\end{aligned}
$$


followed by

$$
\begin{aligned}
d_{11} & =-\tilde{d}_{10}+\tilde{d}_{11}-\tilde{d}_{01}+\tilde{d}_{00}-b_{1 \ell} \Phi_{\ell 1}^{R} \tilde{b}_{00}-\tilde{c}_{00} \Phi_{1 \ell}^{L} c_{\ell 1}-2 \tilde{c}_{00} \tilde{b}_{00} \\
d_{1 j} & =\tilde{d}_{1 j}-\tilde{d}_{0 j}-\tilde{c}_{00} \Phi_{1 \ell}^{L} c_{\ell j} \\
d_{j 1} & =\tilde{d}_{j 1}-\tilde{d}_{j 0}-b_{j \ell} \Phi_{\ell 1}^{R} \tilde{b}_{00} \\
d_{j_{1} j_{2}} & =\tilde{d}_{j_{1} j_{2}} \\
e_{1} & =-\tilde{d}_{01}+\tilde{e}_{0}+\tilde{e}_{1}-\tilde{c}_{00} \Phi_{1 \ell}^{L} c_{\ell 1}-\tilde{c}_{00} \tilde{b}_{00}+\left(\tilde{e}_{0}-\tilde{d}_{00}\right) \phi_{B}\left(a_{10}\right)+\phi_{B}\left(a_{01}\right) \tilde{e}_{0} \\
e_{j} & =\tilde{e}_{j} \\
d_{00}= & \tilde{d}_{00} \quad e_{0}=\tilde{e}_{0} \quad d_{10}=\tilde{d}_{11}-\tilde{e}_{1} \quad d_{01}=\tilde{e}_{1} \quad d_{j 0}=\tilde{d}_{j 0} \quad d_{0 j}=\tilde{d}_{0 j},
\end{aligned}
$$

and finally

$$
\begin{array}{lll}
b_{10}=-\tilde{b}_{10}+c_{11}+\tilde{c}_{10}-a_{1 \ell} \Phi_{\ell 1}^{R} \tilde{b}_{00} & & c_{01}=-\tilde{c}_{01}+b_{11}+\tilde{b}_{01}-\tilde{c}_{00} \Phi_{1 \ell}^{L} a_{\ell 1} \\
-\left(b_{1 \ell} \Phi_{\ell 1}^{R}+\tilde{b}_{0 \ell} \Phi_{\ell 1}^{R}+2 \tilde{c}_{00}\right) \phi_{B}\left(a_{10}\right) & \text { and } & -\phi_{B}\left(a_{01}\right)\left(\Phi_{1 \ell}^{L} c_{\ell 1}+\Phi_{1 \ell}^{L} \tilde{c}_{\ell 0}+2 \tilde{b}_{00}\right) ; \\
b_{00}=\tilde{b}_{00}+\Phi_{1 \ell}^{L} \tilde{c}_{\ell 0} & \text { and } & c_{00}=\tilde{c}_{00}+\tilde{b}_{0 \ell} \Phi_{\ell 1}^{R} ; \\
b_{j 0}=-\tilde{b}_{j 0}+\tilde{c}_{j 0}+b_{j \ell} \Phi_{\ell 1}^{R}-\Phi_{j \ell}^{L} \tilde{c}_{\ell 0} & \text { and } & c_{0 j}=-\tilde{c}_{0 j}+\tilde{b}_{0 j}+\Phi_{1 \ell}^{L} c_{\ell j}-\tilde{b}_{0 \ell} \Phi_{\ell j}^{R} ; \\
b_{0 i}=\tilde{b}_{0 i} & \text { and } & c_{i 0}=\tilde{c}_{i 0} .
\end{array}
$$

We find that $\partial=\tilde{\partial}$ as before, where we extend $\partial$ by defining $\partial b_{0 i}=a_{0 i}-\Phi_{1 \ell}^{L} a_{\ell i}$, $\partial c_{i 0}=a_{i 0}-a_{i \ell} \Phi_{\ell 1}^{R}, \partial d_{00}=b_{00}-c_{00}, \partial e_{0}=b_{00}, \partial d_{i 0}=-b_{i 0}, \partial d_{0 i}=c_{0 i}$. (The calculation uses the fact that $\Phi_{1 \ell}^{L} a_{\ell m} \Phi_{m 1}^{R}=\left(\phi_{B}(A)\right)_{11}=-2$.) It follows that the knot DGA for $B \sigma_{0}^{-1}$ is tamely isomorphic to a stabilization of the knot DGA for $B$, as desired.

\section{Properties of the invariants}

\subsection{Conjugation and abelianization}

There is a symmetry in the homomorphism $\phi: B_{n} \rightarrow \operatorname{Aut}\left(\mathcal{A}_{n}\right)$ which induces a symmetry in braid and knot DGAs. Define an involution on $\mathcal{A}_{n}$, which we term conjugation and write as $v \mapsto \bar{v}$, as follows: the conjugate of a monomial $a_{i_{1} j_{1}} \cdots a_{i_{m} j_{m}}$ is $a_{j_{m} i_{m}} \cdots a_{j_{1} i_{1}}$; extend conjugation linearly, and set $\overline{1}=1$. In other words, conjugation replaces each generator $a_{i j}$ by $a_{j i}$ and then reverses the order of each word. The key observation is the following.

Proposition 6.1 For all $v \in \mathcal{A}_{n}$ and any $B \in B_{n}$, we have $\phi_{B}(\bar{v})=\overline{\phi_{B}(v)}$. 
Proof The result holds for $B=\sigma_{k}$ and $v=a_{i j}$ for any $i, j, k$, by direct inspection of the definition of $\phi$. The general result for $B=\sigma_{k}$ follows since $\overline{v w}=\bar{w} \bar{v}$ for all $v, w \in \mathcal{A}_{n}$; since $\phi$ is a homomorphism, the entire proposition follows.

One consequence of Proposition 6.1 is that the matrices $\Phi_{B}^{L}(A), \Phi_{B}^{R}(A)$ determine each other. Extend the operation of conjugation to matrices by conjugating each entry, and let ${ }^{T}$ denote transpose; then we have the following result.

Proposition 6.2 For any $B \in B_{n}, \Phi_{B}^{R}(A)={\overline{\Phi_{B}^{L}(A)}}^{T}$.

Proof By Proposition 6.1, we have

$$
\phi_{B}\left(a_{* i}\right)=\phi_{B}\left(\overline{a_{i *}}\right)=\overline{\phi_{B}\left(a_{i *}\right)}=\overline{\sum_{j=1}^{n}\left(\Phi_{B}^{L}(A)\right)_{i j} a_{j *}}=\sum_{j=1}^{n} a_{* j}\left(\overline{\Phi_{B}^{L}(A)}\right)_{i j} ;
$$

now use the definition of $\Phi_{B}^{R}(A)$.

Conjugation also allows us to define simplified commutative versions of the braid and knot DGAs. Intuitively, we can quotient the braid and knot DGAs by conjugation and abelianize; the differential is well-defined on the resulting quotient algebras. For instance, on the braid DGA, if $\partial b_{i j}=a_{i j}-\phi_{B}\left(a_{i j}\right)$, then $\partial b_{j i}=a_{j i}-\phi_{B}\left(a_{j i}\right)=\overline{a_{i j}-\phi_{B}\left(a_{i j}\right)}$, and so $\partial$ is still well-defined if we $\bmod$ out by $a_{j i}=a_{i j}, b_{j i}=b_{i j}$ and then abelianize.

Definition 6.3 Let $B \in B_{n}$ be a braid. Let $\mathcal{A}$ be the graded sign-commutative algebra on $n(n-1)$ generators, $\left\{a_{i j} \mid 1 \leq i<j \leq n\right\}$ of degree 0 and $\left\{b_{i j} \mid 1 \leq i<j \leq n\right\}$ of degree 1 . Define a differential $\partial$ on $\mathcal{A}$ to be the usual differential on the braid DGA, where we set $a_{j i}=a_{i j}$ for $j>i$. Then we call $(\mathcal{A}, \partial)$ the abelian braid $D G A$ of $B$.

Here by "sign-commutative," we mean that $v w=(-1)^{(\operatorname{deg} v)(\operatorname{deg} w)} w v$ for all $v, w \in \mathcal{A}$ of pure degree.

For the knot DGA, extend the conjugation operation to the entire algebra by defining $\overline{b_{i j}}=c_{j i}, \overline{c_{i j}}=b_{j i}, \overline{d_{i j}}=-d_{j i}, \overline{e_{i}}=e_{i}-d_{i i}$, and $\overline{v w}=$ $(-1)^{(\operatorname{deg} v)(\operatorname{deg} w)} \bar{w} \bar{v}$.

Proposition 6.4 If $(\mathcal{A}, \partial)$ is the knot DGA for some braid, then $\overline{\partial v}=\partial \bar{v}$ for all $v \in \mathcal{A}$. 
Proof We calculate directly that

$$
\begin{gathered}
\overline{\partial b_{i j}}=\overline{a_{i j}}-\sum_{\ell} \overline{a_{\ell j}} \overline{\left(\Phi_{B}^{L}(A)\right)_{i \ell}}=\partial c_{j i}, \\
\overline{\partial c_{i j}}=\overline{a_{i j}}-\sum_{\ell} \overline{\left(\Phi_{B}^{R}(A)\right)_{\ell j}} \overline{a_{i \ell}}=\partial b_{j i}, \\
\overline{\partial d_{i j}}=\overline{b_{i j}}-\overline{c_{i j}}+\sum_{\ell}\left(-\overline{\left(\Phi_{B}^{R}(A)\right)_{\ell j}} \overline{b_{i \ell}}+\overline{c_{\ell j}} \overline{\left(\Phi_{B}^{L}(A)\right)_{i \ell}}\right)=-\partial d_{j i}, \\
\overline{\partial e_{i}}=\overline{b_{i i}}+\sum_{\ell} \overline{c_{\ell i}} \overline{\left(\Phi_{B}^{L}(A)\right)_{i \ell}}=c_{i i}+b_{i \ell}\left(\Phi_{B}^{R}(A)\right)_{\ell i}=\partial e_{i}-\partial d_{i i},
\end{gathered}
$$

and if $\overline{\partial v}=\partial \bar{v}$ and $\overline{\partial w}=\partial \bar{w}$, then

$$
\overline{\partial(v w)}=(-1)^{(\operatorname{deg} v-1)(\operatorname{deg} w)} \bar{w} \overline{\partial v}+(-1)^{(\operatorname{deg} v)(\operatorname{deg} w)} \overline{\partial w} \bar{v}=\partial(\overline{v w}) .
$$

The proposition follows.

Since $\partial$ commutes with conjugation, we may mod out by conjugation and abelianize, and $\partial$ is well-defined on the resulting algebra.

Definition 6.5 Let $B \in B_{n}$. Let $\mathcal{A}$ be the graded sign-commutative algebra with generators $\left\{a_{i j} \mid 1 \leq i<j \leq n\right\}$ of degree $0,\left\{b_{i j} \mid 1 \leq i, j \leq n\right\}$ of degree 1 , and $\left\{d_{i j} \mid 1 \leq i<j \leq n\right\}$ and $\left\{e_{i} \mid 1 \leq i \leq n\right\}$ of degree 2. Define $\partial$ on $\mathcal{A}$ to be the usual differential on the knot DGA, where we set $a_{j i}=a_{i j}$ for $j>i$ and $c_{i j}=b_{j i}$ for all $i, j$. Then we call $(\mathcal{A}, \partial)$ the abelian knot $D G A$ of $B$.

The notion of stable tame isomorphism can be defined for polynomial algebras just as for tensor algebras, and with respect to this relation, abelian braid and knot DGAs give invariants.

Proposition 6.6 Up to stable tame isomorphism, the abelian braid DGA of $B$ is an invariant of the conjugacy class of $B$, and the abelian knot DGA of $B$ is an invariant of the knot closure of $B$.

Proof Trace through the invariance proofs for the original knot and braid DGAs, and note that all of the tame isomorphisms and stabilizations used there are invariant under conjugation and hence well-defined in the commutative case.

As in the noncommutative case, our main interest in the abelian DGAs lies in the homology in degree 0 , which we write as $H C_{0}^{\mathrm{ab}}(B)$ in the braid case and $H C_{0}^{\mathrm{ab}}(K)$ in the knot case. 
Corollary 6.7 Up to isomorphism, $H C_{0}^{\mathrm{ab}}(B)$ is an invariant of the conjugacy class of $B$, and $H C_{0}^{\mathrm{ab}}(K)$ is an invariant of the knot $K$.

We can summarize the various flavors of $H C_{0}$ in a diagram, as follows. Suppose that braid $B$ has closure $K$, and let $\mathrm{ab}\left(H C_{0}(B)\right), \mathrm{ab}\left(H C_{0}(K)\right)$ represent the abelianizations of $H C_{0}(B), H C_{0}(K)$. Then $H C_{0}(B), H C_{0}(K)$ can both be written as $\mathbb{Z}\left\langle\left\{a_{i j}\right\}\right\rangle$ modulo some ideal, and the ideal for $H C_{0}(K)$ contains the ideal for $H C_{0}(B)$ by Proposition 4.8. Also, ab $\left(H C_{0}(B)\right), H C_{0}^{\mathrm{ab}}(B)$ are both $\mathbb{Z}\left[\left\{a_{i j}\right\}\right]$ modulo some ideal, and the ideal for $H C_{0}^{\mathrm{ab}}(B)$ is generated by the ideal for $\operatorname{ab}\left(H C_{0}(B)\right)$ and the relations $\left\{a_{i j}-a_{j i}\right\}$. Thus we have the following commutative diagram of invariants:

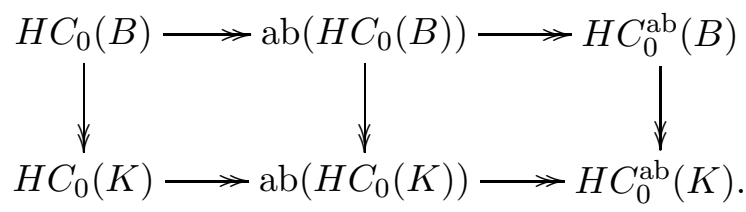

\subsection{Mirrors and inverses}

In this section, we show that the braid and knot DGAs do not change under mirroring and inversion. Recall that the mirror of a knot $K$ is the knot $\bar{K}$ obtained by reversing each of the crossings of the diagram of $K$, and similarly for the mirror $\bar{B}$ of a braid $B$. In $B_{n}$, mirroring is the homomorphism sending $\sigma_{k}$ to $\sigma_{k}^{-1}$ for each $k$. It will be convenient for us to consider a related mirror operation on $B_{n}, B \mapsto B^{*}$, which is the homomorphism sending $\sigma_{k}$ to $\sigma_{n-k}^{-1}$ for each $k$. Recall also that the inverse of a knot is the knot with the opposite orientation. If $K$ is the closure of $B \in B_{n}$, then the mirror $\bar{K}$ is the closure of both $\bar{B}$ and $B^{*}$, while the inverse of $K$ is the closure of $\bar{B}^{-1}$.

Proposition 6.8 The braid DGAs for a braid $B$, its mirrors $\bar{B}$ and $B^{*}$, and its inverse $B^{-1}$ are stable tame isomorphic.

Proof Let $B \in B_{n}$. We first show that $B$ and $B^{-1}$ have equivalent braid DGAs. In the braid DGA of $B^{-1}$, we have $\partial b_{i j}=a_{i j}-\phi_{B^{-1}}\left(a_{i j}\right)$ for all $i, j$; hence if we replace $a_{i j}$ by $\phi_{B}\left(a_{i j}\right)$ and $b_{i j}$ by $-b_{i j}$ for all $i, j$, then we obtain $\partial b_{i j}=a_{i j}-\phi_{B}\left(a_{i j}\right)$, which gives the braid DGA for $B$.

Next, as in [1], denote $\Delta=\left(\sigma_{1} \cdots \sigma_{n-1}\right)\left(\sigma_{1} \cdots \sigma_{n-2}\right) \cdots\left(\sigma_{1} \sigma_{2}\right) \sigma_{1}$. Then $\sigma_{n-k}=$ $\Delta \sigma_{k} \Delta^{-1}$ for all $k$, and so $\bar{B}$ and $B^{*}$ are conjugate for any $B \in B_{n}$; hence by Theorem [2.7] the braid DGAs of $\bar{B}$ and $B^{*}$ are stable tame isomorphic. 
It now suffices to show that $B$ and $B^{*}$ have equivalent braid DGAs. Consider the tame automorphism $\xi$ of the algebra $\mathcal{A}_{n}$ which sends $a_{i j}$ to $a_{n+1-i, n+1-j}$ for each generator $a_{i j}$ (and sends 1 to 1). By inspecting the definition of $\phi$, we see that the induced action of $\xi$ sends $\phi_{\sigma_{k}}$ to $\phi_{\sigma_{n-k}^{-1}}$, ie, $\xi \phi_{\sigma_{k}}=\phi_{\sigma_{n-k}^{-1}} \xi$ for all $k$. Hence $\xi \phi_{B^{*}}=\phi_{B} \xi$ for all $B$.

Write the braid DGAs for $B, B^{*}$ as $(\mathcal{A}, \partial),(\mathcal{A}, \tilde{\partial})$, respectively, and extend $\xi$ to a tame automorphism of $\mathcal{A}$ by sending $b_{i j}$ to $b_{n+1-i, n+1-j}$ for each $b_{i j}$. Then

$$
\partial \xi b_{i j}=\partial b_{n+1-i, n+1-j}=\xi\left(a_{i j}\right)-\phi_{B}\left(\xi\left(a_{i j}\right)\right)=\xi\left(a_{i j}-\phi_{B^{*}}\left(a_{i j}\right)\right)=\xi \tilde{\partial} b_{i j},
$$

and so $\xi$ intertwines the differentials $\partial$ and $\tilde{\partial}$.

Proposition 6.9 The knot DGA classes for a knot, its mirror, and its inverse are the same.

Proof Let $B \in B_{n}$ be a braid whose closure is a knot $K$. We first show that the knot DGAs for $B, B^{*}$ are stable tame isomorphic, which implies that $K$ and its mirror have the same knot DGA class. Denote these knot DGAs by $(\mathcal{A}, \partial),\left(\mathcal{A}, \partial^{\prime}\right)$, respectively.

Let $\Xi$ be the map on $n \times n$ matrices which permutes entries according to the definition $(\Xi(M))_{i j}=M_{n+1-i, n+1-j}$; note that $\Xi$ commutes with matrix multiplication. Define the tame automorphism $\xi$ on $\mathcal{A}$ by its action on generators: $\xi(A)=\Xi(A), \xi(B)=\Xi(B), \xi(C)=\Xi(C), \xi(D)=\Xi(D), \xi\left(e_{i}\right)=e_{n+1-i}$.

Inspection of the definitions of $\phi$ and $\Phi^{L}$ shows that $\xi\left(\Phi_{\sigma_{k}}^{L}(A)\right)=\Xi\left(\Phi_{\sigma_{n-k}^{-1}}^{L}(A)\right)$ for all $k$. This, along with Proposition 4.4 and the identity $\xi \phi_{B^{*}} \stackrel{n-k}{=} \phi_{B} \xi$ from the proof of Proposition 6.8 allows us to prove readily by induction that $\xi\left(\Phi_{B^{*}}^{L}(A)\right)=\Xi\left(\Phi_{B}^{L}(A)\right)$. Similarly, we can establish that $\xi\left(\Phi_{B^{*}}^{R}(A)\right)=$ $\Xi\left(\Phi_{B}^{R}(A)\right)$.

Hence

$$
\partial \xi B=\Xi \partial B=\left(1-\Xi\left(\Phi_{B}^{L}(A)\right)\right) \cdot \Xi(A)=\left(1-\xi\left(\Phi_{B^{*}}^{L}(A)\right)\right) \cdot \xi(A)=\xi \partial^{\prime} B
$$

and similarly $\partial \xi C=\xi \partial^{\prime} C$, while

$$
\partial \xi D=\Xi \partial D=\xi(B) \cdot\left(1-\xi\left(\Phi_{B^{*}}^{R}(A)\right)\right)-\left(1-\xi\left(\Phi_{B^{*}}^{L}(A)\right)\right) \cdot \xi(C)=\xi \partial^{\prime} D
$$

and

$\partial \xi e_{i}=\partial e_{n+1-i}=\left(\Xi\left(B+\Phi_{B}^{L}(A) \cdot C\right)\right)_{i i}=\left(\xi(B)+\xi\left(\Phi_{B^{*}}^{L}(A)\right) \cdot \xi(C)\right)_{i i}=\xi \partial^{\prime} e_{i}$.

It follows that $\xi$ intertwines $\partial$ and $\partial^{\prime}$, and so the knot DGAs of $B$ and $B^{*}$ are equivalent. 
Now we show that the knot DGAs of $B$ and $B^{-1}$ are equivalent; this implies that $K$ and its inverse have the same knot DGA class, since $B^{-1},\left(B^{-1}\right)^{*}$ have equivalent knot DGAs by the above argument, and $\left(B^{-1}\right)^{*}, \bar{B}^{-1}$ are conjugate and hence have equivalent knot DGAs by Theorem 2.10, Write the knot DGA of $B^{-1}$ as $(\tilde{\mathcal{A}}, \tilde{\partial})$, where the generators of $\tilde{\mathcal{A}}$ are marked by tildes. Identify the generators of $\tilde{\mathcal{A}}$ with those of $\mathcal{A}$ by $\tilde{A}=\phi_{B}(A), \tilde{B}=-B \cdot \Phi_{B}^{R}(A), \tilde{C}=$ $-\Phi_{B}^{L}(A) \cdot C, \tilde{D}=D$, and $\tilde{e}_{i}=d_{i i}-e_{i}$. Using Corollary 4.5 and Proposition 4.7. we calculate that

$$
\tilde{\partial} \tilde{B}=\left(1-\Phi_{B^{-1}}^{L}(\tilde{A})\right) \cdot \tilde{A}=\left(1-\left(\Phi_{B}^{L}(A)\right)^{-1}\right) \cdot \Phi_{B}^{L}(A) \cdot A \cdot \Phi_{B}^{R}(A)=\partial \tilde{B}
$$

and similarly $\tilde{\partial} \tilde{C}=\partial \tilde{C}$, while

$\tilde{\partial} \tilde{D}=\tilde{B} \cdot\left(1-\Phi_{B^{-1}}^{R}(\tilde{A})\right)-\left(1-\Phi_{B^{-1}}^{L}(\tilde{A})\right) \cdot \tilde{C}=B \cdot\left(1-\Phi_{B}^{R}(A)\right)-\left(1-\Phi_{B}^{L}(A)\right) \cdot C=\partial \tilde{D}$

and

$$
\tilde{\partial} \tilde{e}_{i}=\left(\tilde{B}+\Phi_{B^{-1}}^{L}(\tilde{A}) \cdot \tilde{C}\right)_{i i}=\left(-B \cdot \Phi_{B}^{R}(A)-C\right)_{i i}=\partial\left(d_{i i}-e_{i}\right)=\partial \tilde{e}_{i} .
$$

Hence our identification of generators, which yields a tame automorphism between $\mathcal{A}$ and $\tilde{\mathcal{A}}$, gives $\partial=\tilde{\partial}$, and so the knot DGAs of $B$ and $B^{-1}$ are equivalent.

It can similarly be shown that the abelian braid and knot DGAs do not distinguish between mirrors or inverses. We may then ask how our invariants relate to classical invariants which also fail to distinguish between mirrors or inverses, such as the Alexander polynomial and the absolute value of the signature. In Section [7.1, we establish a connection between the knot DGA and the Alexander polynomial. First, in the next section, we deduce an invariant from the DGAs which shows that our invariants can distinguish between knots with the same Alexander polynomial and signature.

\subsection{Augmentation number}

We now introduce a family of easily computable invariants derived from the braid and knot DGAs. These invariants, which we call augmentation numbers, gives a crude but effective way to distinguish between braid conjugacy classes or knots. The advantage of augmentation numbers is that, unlike $H C_{0}$ or the full DGA, they are positive integers and hence require no ad hoc methods to interpret (cf the computations of $H C_{0}$ in Section 8 ). 
Definition 6.10 If $R$ is a finitely generated ring and $d \geq 2$ an integer, define the $\mathbb{Z}_{d}$ augmentation number of $R$ to be the number of ring homomorphisms $R \rightarrow \mathbb{Z}_{d}$. For $B$ any braid and $K$ any knot, write $\operatorname{Aug}(B, d)$, $\operatorname{Aug}^{\mathrm{ab}}(B, d), \operatorname{Aug}(K, d), \operatorname{Aug}^{\mathrm{ab}}(K, d)$ for the $\mathbb{Z}_{d}$ augmentation numbers of $H C_{0}(B), H C_{0}^{\mathrm{ab}}(B), H C_{0}(K), H C_{0}^{\mathrm{ab}}(K)$, respectively.

We use the term "augmentation" in keeping with [8, in which the case $d=2$ is discussed in the context of DGAs and Poincaré polynomials. One could imagine replacing $\mathbb{Z}_{d}$ by any finite ring, although the usefulness of the more general construction is unclear.

Calculating augmentation numbers is straightforward. For instance, to compute $\operatorname{Aug}(B, d)$ for $B \in B_{n}$, write $H C_{0}(B)=\mathbb{Z}\left\langle\left\{a_{i j}\right\}\right\rangle / \mathcal{I}$ and count the number of points in $\mathbb{Z}_{d}^{n(n-1)}$ (the space parametrizing ring homomorphisms $\mathbb{Z}\left\langle\left\{a_{i j}\right\}\right\rangle \rightarrow$ $\left.\mathbb{Z}_{d}\right)$ which lie in the vanishing set of $\mathcal{I}$. There are $d^{n(n-1)}$ possible points and $n(n-1)$ conditions to check, corresponding to $\partial b_{i j}$ for $1 \leq i \neq j \leq n$. Similarly, for $\operatorname{Aug}^{\mathrm{ab}}(B, d)$, there are $d^{n(n-1) / 2}$ possible points and $n(n-1) / 2$ conditions; for $\operatorname{Aug}(K, d)$ with $K$ the closure of $B, d^{n(n-1)}$ points and $2 n^{2}$ conditions; and for $\operatorname{Aug}^{\mathrm{ab}}(K, d), d^{n(n-1) / 2}$ points and $n^{2}$ conditions. (Note thus that $\mathrm{Aug}^{\mathrm{ab}}$ typically takes considerably less computing time than Aug to calculate.) In all cases, the $\mathbb{Z}_{d}$ augmentation number is at least 1 , as the following result shows.

Proposition 6.11 For any braid $B$ or knot $K$, the ring homomorphism from $\mathcal{A}_{n}$ to $\mathbb{Z}$ defined by sending each generator $a_{i j}$ to -2 descends to homomorphisms from $H C_{0}(B), H C_{0}^{\mathrm{ab}}(B), H C_{0}(K), H C_{0}^{\mathrm{ab}}(K)$ to $\mathbb{Z}$.

Proof It suffices to prove the assertion for $H C_{0}^{\mathrm{ab}}(K)$, where $K$ is the closure of the braid $B$; see the diagram at the end of Section 6.1. Let $M$ denote the $n \times n$ matrix all of whose entries are -2 ; then we need to show that $M=\Phi_{B}^{L}(M) \cdot M$. It is clear from the definition of $\phi$ and Lemma 4.6 that $\phi_{\sigma_{k}}(M)=M$ and $\Phi_{\sigma_{k}}^{L}(M) \cdot M=M$. By Proposition 4.4 it follows that

$$
\Phi_{\sigma_{k} B}^{L}(M) \cdot M=\Phi_{B}^{L}\left(\phi_{\sigma_{k}}(M)\right) \cdot \Phi_{\sigma_{k}}^{L}(M) \cdot M=\Phi_{B}^{L}(M) \cdot M
$$

for any $B$, and hence $\Phi_{B}^{L}(M) \cdot M=M$ for all $B$.

As an example of the computation of augmentation numbers, consider the trefoil $3_{1}$, for which we calculated in Section 4.1 that $H C_{0} \cong \mathbb{Z}[x] /((x+2)(x-1))$; it is also easy to see that $H C_{0}^{\mathrm{ab}}\left(3_{1}\right) \cong \mathbb{Z}[x] /((x+2)(x-1))$. Then $\operatorname{Aug}\left(3_{1}, d\right)=$ $\operatorname{Aug}^{\mathrm{ab}}\left(3_{1}, d\right)=2$ for $d \neq 3$ and $\operatorname{Aug}\left(3_{1}, 3\right)=\operatorname{Aug}^{\mathrm{ab}}\left(3_{1}, 3\right)=1$. Note that these 
augmentation numbers could be calculated without a nice form for $H C_{0}$, by directly using the definition of $\partial$.

Any knot $K$ with $H C_{0}(K) \cong \mathbb{Z}[x] /(p(x))$ for some polynomial $p(x)$ satisfies $\operatorname{Aug}^{\mathrm{ab}}(K, d) \leq \operatorname{Aug}(K, d) \leq d$ for all $d$. Hence augmentation numbers can be used to show that some knots have $H C_{0}$ which is not of the form $\mathbb{Z}[x] /(p(x))$; see the examples in Sections 8.5 and 8.6 .

\section{Knot contact homology and the determinant}

For knots $K$, contact homology is closely related to the classical invariant $\left|\Delta_{K}(-1)\right|$, the determinant of $K$. Throughout this section, we will denote by $\Sigma_{2}=\Sigma_{2}(K)$ the double branched cover of $S^{3}$ over $K$, as in [22]; it is well known that $\left|H_{1}\left(\Sigma_{2}(K)\right)\right|=\left|\Delta_{K}(-1)\right|$. In Section [7.1] we establish a surjection from $H C_{0}(K)$ to a quotient of the polynomial ring $\mathbb{Z}[x]$ specified by the largest invariant factor of $H_{1}\left(\Sigma_{2}(K)\right)$, which typically is equal to the determinant of $K$. In Section [7.2, we discuss a natural linearization of knot contact homology, and see that $H_{1}\left(\Sigma_{2}(K)\right)$, and hence the determinant, can be deduced from this linearization.

\section{1 $H C_{0}(K)$ and $\Sigma_{2}(K)$}

Before stating the main result of this section, we need to introduce a sequence of polynomials which plays a key role in the remainder of the paper. Define $\left\{p_{m} \in\right.$ $\mathbb{Z}[x]\}$ inductively by $p_{0}(x)=2-x, p_{1}(x)=x-2, p_{m+1}(x)=x p_{m}(x)-p_{m-1}(x)$. Perhaps a more illuminating way to view $p_{m}, m \geq 1$, is via its roots:

$$
p_{m}(x)=\prod_{k=0}^{m-1}\left(x-2 \cos \frac{2 k \pi}{2 m-1}\right) \text {. }
$$

Theorem 7.1 For $K$ a knot, let $n(K)$ denote the largest invariant factor of the abelian group $H_{1}\left(\Sigma_{2}(K)\right)$. Then there is a surjective ring homomorphism

$$
H C_{0}(K) \rightarrow \mathbb{Z}[x] /\left(p_{(n(K)+1) / 2}(x)\right),
$$

and this map factors through $H C_{0}^{\mathrm{ab}}(K)$. In particular, the ranks of $H C_{0}(K)$ and $H C_{0}^{\mathrm{ab}}(K)$ are at least $(n(K)+1) / 2$. 
Note that $n(K)$ is odd since the determinant of a knot is always odd, and that $n(K)$ divides $\left|\Delta_{K}(-1)\right|$ and is divisible by any prime dividing $\left|\Delta_{K}(-1)\right|$. A table of invariant factors of $H_{1}\left(\Sigma_{2}(K)\right)$ for small knots can be found in [2].

To prove Theorem 7.1 we will need a number of preliminary results. Define another sequence $\left\{q_{m} \in \mathbb{Z}[x]\right\}$ by $q_{0}(x)=-2, q_{1}(x)=-x, q_{m+1}(x)=$ $x q_{m}(x)-q_{m-1}(x)$. Note that the recursion can be used to define $q_{m}$ for all $m \in \mathbb{Z}$, and that $q_{-m}=q_{m}$ for all $m$ because of symmetry.

Lemma 7.2 For all $m_{1}, m_{2} \in \mathbb{Z}$, we have

$$
q_{m_{1}}(x) q_{m_{2}}(x)+q_{m_{1}+m_{2}}(x)+q_{m_{1}-m_{2}}(x)=0 .
$$

Proof The identity holds by the defining relations for $q_{m}$ for $m_{2}=0$ and $m_{2}=1$. It is then easy to establish by induction that it holds for all $m_{2} \geq 0$, and hence for all $m_{2}$ since $q_{-m_{2}}=q_{m_{2}}$.

Lemma 7.3 For all $\ell, m \in \mathbb{Z}$ with $m \geq 0$, we have $p_{m}(x) \mid q_{\ell+2 m-1}(x)-q_{\ell}(x)$.

Proof An easy induction shows that $\left(p_{m}(x)\right)^{2}=(x+2)\left(q_{2 m-1}(x)+2\right)$ and $p_{m}(x) p_{m+1}(x)=-(x+2)\left(q_{2 m}(x)-x\right)$ for all $m \geq 0$; since $x+2$ divides $p_{m}(x), p_{m+1}(x)$, this establishes the lemma for $\ell=0,1$. The lemma now follows by induction on $\ell$.

One can more elegantly prove Lemmas $\mathbf{7 . 2}$ and 7.3 by noting that the polynomials $p_{m}, q_{m}$ are characterized by the identities $p_{m}\left(x+x^{-1}\right)=x^{m}+x^{-m}-$ $x^{m-1}-x^{1-m}, q_{m}\left(x+x^{-1}\right)=-x^{m}-x^{-m}$; the lemmas then follow easily.

We next need to recall the Burau representation on $B_{n}$; see [1] as a reference. For $1 \leq k \leq n-1$, define Bur $_{\sigma_{k}}$ to be the linear map on the free $\mathbb{Z}\left[t, t^{-1}\right]$ module on $n$ generators whose matrix is the identity except for the $2 \times 2$ submatrix formed by the $k, k+1$ rows and columns, which is $\left(\begin{array}{cc}1-t & t \\ 1 & 0\end{array}\right)$. This extends to a representation of $B_{n}$, classically known as the (nonreduced) Burau representation. The Burau representation is reducible to a trivial 1-dimensional representation spanned by the vector $(1, \ldots, 1)$, and the $(n-1)$-dimensional reduced Burau representation. More concretely, the map $\mathbb{Z}\left[t, t^{-1}\right]^{n} \rightarrow \mathbb{Z}\left[t, t^{-1}\right]^{n-1}$ given by $\left(v_{1}, \ldots, v_{n}\right) \mapsto\left(v_{2}-v_{1}, \ldots, v_{n}-v_{1}\right)$ induces the reduced Burau representation on $\mathbb{Z}\left[t, t^{-1}\right]^{n-1}$.

For the remainder of this section, we will set $t=-1$; then the nonreduced and reduced Burau representations of $B_{n}$ act on $\mathbb{Z}^{n}$ and $\mathbb{Z}^{n-1}$, respectively. Let 
$\operatorname{Bur}_{B}$ and $\operatorname{Bur}_{B}^{0}$ denote the matrices for $B$ in the two respective representations. It is well known that $\operatorname{Bur}_{B}^{0}$ is a presentation matrix for the group $H_{1}\left(\Sigma_{2}(K)\right)$.

We need a bit more notation. If $B$ is a braid, let $\hat{B}$ denote the "reverse" braid obtained by reversing the word which gives $B$; that is, if $B=\sigma_{i_{1}}^{j_{1}} \sigma_{i_{2}}^{j_{2}} \cdots \sigma_{i_{m}}^{j_{m}}$, then $\hat{B}=\sigma_{i_{m}}^{j_{m}} \cdots \sigma_{i_{2}}^{j_{2}} \sigma_{i_{1}}^{j_{1}}$. (In our notation from Section 6.2, $\hat{B}=\bar{B}^{-1}$.) Also, for $v, w \in \mathbb{Z}^{n}$, let $M(v, w)$ denote the $n \times n$ matrix defined by $(M(v, w))_{i j}=$ $q_{v_{i}-w_{j}}$.

Lemma 7.4 Let $B \in B_{n}$ and $v \in \mathbb{Z}^{n}$. If we set $A=M(v, v)$, then $\phi_{B}(A)=$ $M\left(\operatorname{Bur}_{\hat{B}} v, \operatorname{Bur}_{\hat{B}} v\right)$.

Proof Since $A=M(v, v)$, we have $a_{i j}=q_{v_{i}-v_{j}}$ for all $i, j$. The key is to verify the relation for $B=\sigma_{k}$. If $i \neq k, k+1$, then

$$
\begin{aligned}
\phi_{\sigma_{k}}\left(a_{k i}\right) & =-a_{k+1, i}-a_{k+1, k} a_{k i}=-q_{v_{k+1}-v_{i}}-q_{v_{k+1}-v_{k}} q_{v_{k}-v_{i}}=q_{2 v_{k}-v_{k+1}-v_{i}} \\
& =\left(M\left(\operatorname{Bur}_{\sigma_{k}} v, \operatorname{Bur}_{\sigma_{k}} v\right)\right)_{k i}
\end{aligned}
$$

by Lemma [7.2 and similarly $\phi_{\sigma_{k}}\left(a_{i k}\right)=\left(M\left(\operatorname{Bur}_{\sigma_{k}} v, \operatorname{Bur}_{\sigma_{k}} v\right)\right)_{i k}$;

$$
\phi_{\sigma_{k}}\left(a_{k+1, i}\right)=a_{k i}=q_{v_{k}-v_{i}}=\left(M\left(\operatorname{Bur}_{\sigma_{k}} v, \operatorname{Bur}_{\sigma_{k}} v\right)\right)_{k+1, i}
$$

and similarly $\phi_{\sigma_{k}}\left(a_{i, k+1}\right)=\left(M\left(\operatorname{Bur}_{\sigma_{k}} v, \operatorname{Bur}_{\sigma_{k}} v\right)\right)_{i, k+1}$;

$$
\phi_{\sigma_{k}}\left(a_{k, k+1}\right)=a_{k+1, k}=q_{v_{k+1}-v_{k}}=q_{v_{k}-v_{k+1}}=\left(M\left(\operatorname{Bur}_{\sigma_{k}} v, \operatorname{Bur}_{\sigma_{k}} v\right)\right)_{k, k+1}
$$

and similarly $\phi_{\sigma_{k}}\left(a_{k+1, k}\right)=\left(M\left(\operatorname{Bur}_{\sigma_{k}} v, \operatorname{Bur}_{\sigma_{k}} v\right)\right)_{k+1, k}$; and finally, if $i, j \neq$ $k, k+1$, then $\phi_{\sigma_{k}}\left(a_{i j}\right)=q_{v_{i}-v_{j}}=\left(M\left(\operatorname{Bur}_{\sigma_{k}} v, \operatorname{Bur}_{\sigma_{k}} v\right)\right)_{i j}$. This shows that $\phi_{\sigma_{k}}(A)=M\left(\operatorname{Bur}_{\sigma_{k}} v, \operatorname{Bur}_{\sigma_{k}} v\right)$.

By replacing $v$ by $\operatorname{Bur}_{\sigma_{k}^{-1}} v$, we can deduce the desired identity for $B=\sigma_{k}^{-1}$ from the identity for $B=\sigma_{k}$. It thus suffices to show that if the identity holds for $B=B_{1}$ and $B=B_{2}$, then it holds for $B=B_{1} B_{2}$. Now

$$
\begin{aligned}
\phi_{B_{1} B_{2}}(M(v, v)) & =\phi_{B_{1}}\left(\phi_{B_{2}}(M(v, v))\right) \\
& =\phi_{B_{2}}\left(M\left(\operatorname{Bur}_{\hat{B_{1}}} v, \operatorname{Bur}_{\hat{B_{1}}} v\right)\right) \\
& =M\left(\operatorname{Bur}_{\hat{B_{2}}} \operatorname{Bur}_{\hat{B_{1}}} v, \operatorname{Bur}_{\hat{B_{2}}} \operatorname{Bur}_{\hat{B_{1}}} v\right) \\
& =M\left(\operatorname{Bur}_{\widehat{B_{1} B_{2}}} v, \operatorname{Bur}_{\widehat{B_{1} B_{2}}} v\right),
\end{aligned}
$$

and the lemma follows.

Lemma 7.5 Let $B \in B_{n}$ and $v \in \mathbb{Z}^{n}$. If $A=M(v, v)$, then $\Phi_{B}^{L}(A) \cdot A=$ $M\left(\operatorname{Bur}_{\hat{B}} v, v\right)$ and $A \cdot \Phi_{B}^{R}(A)=M\left(v, \operatorname{Bur}_{\hat{B}} v\right)$. 
Proof We will prove the identity involving $\Phi^{L}$; the proof for $\Phi^{R}$ is completely analogous. First note that the identity is obvious when $B$ is trivial. Now suppose that the identity holds for $B$; we show that it holds for $B \sigma_{k}$. Indeed, by Proposition 4.4, we have

$$
\Phi_{B \sigma_{k}}^{L}(A) \cdot A=\Phi_{\sigma_{k}}^{L}\left(\phi_{B}(A)\right) \cdot \Phi_{B}^{L}(A) \cdot A=\Phi_{\sigma_{k}}^{L}\left(\phi_{B}(A)\right) \cdot M\left(\operatorname{Bur}_{\hat{B}} v, v\right) .
$$

By Lemma 4.6. $\left(\Phi_{B \sigma_{k}}^{L}(A) \cdot A\right)_{i j}=\left(\operatorname{Bur}_{\hat{B}} v\right)_{i}-v_{j}$ except when $i=k$ or $i=k+1$; in these cases, Lemmas 4.6, 7.2, and 7.4 yield

$$
\begin{aligned}
\left(\Phi_{B \sigma_{k}}^{L}(A) \cdot A\right)_{k j} & =-\phi_{B}\left(a_{k+1, k}\right)\left(M\left(\operatorname{Bur}_{\hat{B}} v, v\right)\right)_{k j}-\left(M\left(\operatorname{Bur}_{\hat{B}} v, v\right)\right)_{k+1, j} \\
& =-\left(q_{\left(\operatorname{Bur}_{\hat{B}} v\right)_{k+1}-\left(\operatorname{Bur}_{\hat{B}} v\right)_{k}}\right)\left(q_{\left(\operatorname{Bur}_{\hat{B}} v\right)_{k}-v_{j}}\right)-q_{\left(\operatorname{Bur}_{\hat{B}} v\right)_{k+1}-v_{j}} \\
& =q_{\left(2\left(\operatorname{Bur}_{\hat{B}} v\right)_{k}-\left(\operatorname{Bur}_{\hat{B}} v\right)_{k+1}-v_{j}\right)}, \\
\left(\Phi_{B \sigma_{k}}^{L}(A) \cdot A\right)_{k+1, j} & =\left(M\left(\operatorname{Bur}_{\hat{B}} v, v\right)\right)_{k j}=q_{\left(\operatorname{Bur}_{\hat{B}} v\right)_{k}-v_{j}} .
\end{aligned}
$$

In all cases, we have $\left(\Phi_{B \sigma_{k}}^{L}(A) \cdot A\right)_{i j}=q_{\left(\operatorname{Bur}_{\sigma_{k} \hat{B}} v\right)_{i}-v_{j}}$, and so the identity holds for $B \sigma_{k}$. A similar computation shows that if the identity holds for $B$, then it holds for $B \sigma_{k}^{-1}$. The identity for all $B$ follows by induction.

Proof of Theorem 7.1 Since $\operatorname{Bur}_{\hat{B}}^{0}$ is a presentation matrix for $H_{1}\left(\Sigma_{2}(K)\right)=$ $H_{1}\left(\Sigma_{2}(\bar{K})\right)$, and $n(K)$ is by definition an invariant factor of $H_{1}\left(\Sigma_{2}(K)\right)$, there exists a vector $v=\left(v_{2}, \ldots, v_{n}\right) \in \mathbb{Z}^{n-1}$ with $\operatorname{gcd}\left(v_{2}, \ldots, v_{n}, n(K)\right)=1$, such that the vector $\left(\operatorname{Bur}_{\hat{B}}^{0}-1\right) v$ is divisible by $n(K)$. This means that $v^{\prime}=$ $\left(0, v_{2}, \ldots, v_{n}\right) \in \mathbb{Z}^{n}$ satisfies the following property: if we write $w=\left(\operatorname{Bur}_{\hat{B}}-\right.$ 1) $v^{\prime}$, then $w_{i} \equiv w_{j}(\bmod n(K))$ for all $1 \leq i, j \leq n$. Since the Burau representation sends $(1, \ldots, 1)$ to itself, we may define $v^{\prime \prime}=\left(-w_{1}, v_{2}-w_{1}, \ldots, v_{n}-w_{1}\right)$ and conclude that the vector $\left(\operatorname{Bur}_{\hat{B}}-1\right) v^{\prime \prime}$ is divisible by $n(K)$.

Now consider the ring homomorphism $\rho: \mathcal{A}_{n} \rightarrow \mathbb{Z}[x]$ defined by sending the matrix $A$ to $M\left(v^{\prime \prime}, v^{\prime \prime}\right)$. By Lemma[7.5 $\rho$ sends $\left(1-\Phi_{B}^{L}(A)\right) \cdot A$ to $M\left(v^{\prime \prime}, v^{\prime \prime}\right)-$ $M\left(\operatorname{Bur}_{\hat{B}} v^{\prime \prime}, v^{\prime \prime}\right)$. Since $\operatorname{Bur}_{\hat{B}} v^{\prime \prime} \equiv v^{\prime \prime}(\bmod n(K))$, Lemma 7.3 shows that for all $i, j$,

$$
p_{(n(K)+1) / 2} \mid q_{v_{i}^{\prime \prime}-v_{j}^{\prime \prime}}-q_{\left(\operatorname{Bur}_{\hat{B}} v^{\prime \prime}\right)_{i}-v_{j}^{\prime \prime}}=\left(M\left(v^{\prime \prime}, v^{\prime \prime}\right)-M\left(\operatorname{Bur}_{\hat{B}} v^{\prime \prime}, v^{\prime \prime}\right)\right)_{i j} .
$$

Hence $p_{(n(K)+1) / 2}$ divides all entries of $\rho\left(\left(1-\Phi_{B}^{L}(A)\right) \cdot A\right)$. Similarly, $p_{(n(K)+1) / 2}$ divides all entries of $\rho\left(A \cdot\left(1-\Phi_{B}^{R}(A)\right)\right)$. It follows that $\rho$ descends to a map $\tilde{\rho}: H C_{0}(K) \rightarrow \mathbb{Z}[x] /\left(p_{(n(K)+1) / 2}(x)\right)$, which clearly factors through $H C_{0}^{\mathrm{ab}}(K)$ since $q_{m}=q_{-m}$.

It remains to show that $\tilde{\rho}$ is surjective, or equivalently, that $x$ is in the image of $\tilde{\rho}$. Define the set $S=\left\{j \in \mathbb{Z} \mid q_{j}(x) \in \operatorname{im} \rho\right\}$. We first claim that $S=g \mathbb{Z}$ for some $g \in \mathbb{Z}$. 
By Lemma 7.2, if $m_{1} \in S$, then $m_{1}+m_{2} \in S$ if and only if $m_{1}-m_{2} \in S$. Hence if $a_{1}, a_{2} \in S$, then the arithmetic progression $\left\{a_{1}+\ell\left(a_{2}-a_{1}\right)\right\}_{\ell \in \mathbb{Z}}$ is contained in $S$. Now suppose that $\left(m_{1}, m_{2}\right)=\left(a_{1}, a_{2}\right)$ minimizes $\left|m_{1}-m_{2}\right|$, where $\left(m_{1}, m_{2}\right)$ ranges over all pairs of distinct elements in $S$. Then $S=\left\{a_{1}+\ell\left(a_{2}-a_{1}\right)\right\}_{\ell \in \mathbb{Z}}$, since the existence of an element of $S$ not in the arithmetic progression would violate minimality. Furthermore, $0 \in S$ since $q_{0}(x)=-2 \in \operatorname{im} \rho$. Hence $S$ is an ideal in $\mathbb{Z}$, which proves the claim.

Now for $2 \leq i \leq n$, we have $\rho\left(a_{i 1}\right)=q_{v_{i}^{\prime \prime}-v_{1}^{\prime \prime}}=q_{v_{i}}$, and so $v_{i} \in S$. By the claim, we conclude that all multiples of $\operatorname{gcd}\left(v_{2}, \ldots, v_{n}\right)$ are in $S$. Since $\operatorname{gcd}\left(v_{2}, \ldots, v_{n}\right)$ and $n(K)$ are relatively prime by construction, there exists $j \in S$ with $j \equiv 1$ $(\bmod n(K))$. By Lemmal7.3 $p_{(n(K)+1) / 2}$ divides $q_{j}(x)-q_{1}(x) ;$ but $q_{j}(x) \in \operatorname{im} \rho$ and $q_{1}(x)=-x$, and hence $x \in \operatorname{im} \tilde{\rho}$, as desired.

The above proof is a bit tedious and hard to digest. In [19], we will use the cord ring to see the surjection from Theorem 7.1 rather more directly, and justify the appearance of the polynomials $p_{m}$.

\subsection{Linearized knot contact homology}

Although it is generally difficult to find a nice form for the full knot contact homology $H C_{*}(K)$, there is a canonical linearization of $H C_{*}(K)$ which is easy to compute, and which encodes the group $H_{1}\left(\Sigma_{2}(K)\right)$ and hence the determinant of $K$. We will discuss this linearization in this section.

Let $(\mathcal{A}, \partial)$ be the knot DGA for a knot $K$. It follows easily from Proposition 6.11 that the algebra map $\epsilon: \mathcal{A} \rightarrow \mathbb{Z}$, sending each generator $a_{i j}$ to -2 , and all other generators to 0 , gives an augmentation for $(A, \partial)$; that is, $\epsilon \circ \partial=0$. As in [3], we can construct linearized homology groups via this augmentation.

Let $\varphi_{\epsilon}: \mathcal{A} \rightarrow \mathcal{A}$ be the algebra isomorphism which sends $a_{i j}$ to $a_{i j}-2$ for all $i, j$, and acts as the identity on the other generators $b_{i j}, c_{i j}, d_{i j}, e_{i}$. Let $\mathcal{M}$ be the subalgebra of $\mathcal{A}$ generated by all words of length at least 1 . Then $\partial_{\epsilon}:=\varphi_{\epsilon} \circ \partial \circ \varphi_{\epsilon}^{-1}$ maps $\mathcal{M}$ into itself. For any $k \geq 2, \partial_{\epsilon}$ descends to a differential $\partial_{\epsilon}^{\text {lin }}$ on the finite-rank graded $\mathbb{Z}$-module $\mathcal{M} / \mathcal{M}^{k}$, and the homology of the resulting complex gives a linearized version of $H C_{*}(K)$.

We now restrict our attention to the simplest nontrivial case, $k=2$, although higher values of $k$ also yield knot invariants. Note that $\mathcal{M} / \mathcal{M}^{2}$ is a free $\mathbb{Z}$ module generated in degree 0 by $a_{i j}$, in degree 1 by $b_{i j}$ and $c_{i j}$, and in degree 2 by $d_{i j}$ and $e_{i}$. 
Definition 7.6 The linearized contact homology of $K$, written $H C_{*}^{\operatorname{lin}}(K)$, is the graded homology of $\partial_{\epsilon}$ on $\mathcal{M} / \mathcal{M}^{2}$.

Using the conjugation symmetry of Section 6.1 we can also define an abelian version of linearized contact homology. In this case, $(\mathcal{A}, \partial)$ is the abelian knot DGA of $K$ with corresponding maximal subalgebra $\mathcal{M}$, and $\partial_{\epsilon}$ descends to a differential on $\mathcal{M} / \mathcal{M}^{2}$, whose homology we denote by $H C_{*}^{\mathrm{ab}, \operatorname{lin}}(K)$.

Proposition 7.7 The groups $H C_{*}^{\operatorname{lin}}(K)$ and $H C_{*}^{\text {ab,lin }}(K)$ are invariants of the knot $K$.

Proof All of the stable tame isomorphisms used in the proof of Theorem 2.10 commute with $\epsilon$, because $\phi_{B} \circ \epsilon=\epsilon \circ \phi_{B}$ for any braid $B$. The proposition follows.

We now establish some results about $H C_{*}^{\operatorname{lin}}(K)$. Note that this homology exists only in dimensions $*=0,1,2$.

Proposition 7.8 For $K$ a knot, $H C_{2}^{\operatorname{lin}}(K) \cong \mathbb{Z}$ and $H C_{1}^{\operatorname{lin}}(K) \cong\left(H_{1}\left(\Sigma_{2}\right) \oplus\right.$ $\mathbb{Z}) \otimes\left(H_{1}\left(\Sigma_{2}\right) \oplus \mathbb{Z}\right) \oplus \mathbb{Z}^{m}$ for some $m \geq 0$.

Proposition 7.8 will follow almost immediately from a lemma which we now formulate. Let $d_{1}, d_{2}, \ldots, d_{k}$ be the invariant factors of $H_{1}\left(\Sigma_{2}\right)$, with $d_{1}\left|d_{2}\right| \cdots \mid d_{k}$. Write $Y=\operatorname{Bur}_{\hat{B}}$, and express $1-Y$ in Smith normal form; more precisely, there are matrices $X_{1}, X_{2} \in G L(n, \mathbb{Z})$ with $X_{1} \cdot(1-Y) X_{2}=\Delta$, where $\Delta$ is a diagonal matrix. Since $1-Y$ is a presentation matrix for $H_{1}\left(\Sigma_{2}\right) \oplus \mathbb{Z}$, we may assume that the diagonal entries of $\Delta$ are precisely $d_{1}, \ldots, d_{n}$, where $d_{k+1}=\cdots=d_{n-1}=1$ and $d_{n}=0$.

Now write the linearized chain complex of the alternate knot DGA of the braid $B$ as $C_{2} \stackrel{M_{2}}{\longrightarrow} C_{1} \stackrel{M_{1}}{\longrightarrow} C_{0}$, where $C_{0}, C_{1}, C_{2}$ are generated by $\left\{a_{i j}\right\},\left\{b_{i j}, c_{i j}, d_{i j}\right\}$, $\left\{e_{i j}, f_{i j}\right\}$, respectively. The map $M_{2}$ is given by the action of $\partial_{\epsilon}^{\operatorname{lin}}$ on $C_{2}$ : $\partial_{\epsilon}^{\operatorname{lin}} E=B-D-C \cdot\left(\operatorname{Bur}_{\hat{B}}\right)^{T}, \partial_{\epsilon}^{\operatorname{lin}} F=B-C-\operatorname{Bur}_{\hat{B}} \cdot D$.

Lemma 7.9 After changing bases for $C_{1}$ and $C_{2}$, we can write $M_{2}$ as a diagonal $\left(3 n^{2}-n\right) \times\left(2 n^{2}\right)$ matrix, with $n^{2}$ diagonal entries given by $\operatorname{gcd}\left(d_{i}, d_{j}\right)=$ $d_{\min (i, j)}, 1 \leq i, j \leq n$, and the remaining $n^{2}$ diagonal entries given by 1 . 
Proof We wish to calculate Smith normal form for $M_{2}$. Replace $D$ by $D+$ $B-C \cdot Y^{T}$ and then $F$ by $F+Y \cdot E$ (this simply reparametrizes $d_{i j}$ and $f_{i j}$ ); then $\partial_{\epsilon}^{\operatorname{lin}} E=-D$ and $\partial_{\epsilon}^{\operatorname{lin}} F=B-C-Y \cdot\left(B-C \cdot Y^{T}\right)$. The action of $\partial_{\epsilon}^{\operatorname{lin}}$ on $E$ gives $n^{2}$ "1" diagonal entries in $M_{2}$. To complete the Smith normal form for $M_{2}$, we need to examine the matrix $Z=B-C-Y \cdot\left(B-C \cdot Y^{T}\right)$.

Introduce dummy variables $b_{11}, \ldots, b_{n n}$ (this adds $n$ rows of zeros to $M_{2}$ ), and replace $b_{i j}$ by $b_{i j}-b_{j j}$ for all $i \neq j$. If we write $\tilde{B}=\left(b_{i j}\right)$, which is identical to $B$ away from the diagonal, then $Z$ becomes $\tilde{B}-C-Y \cdot\left(\tilde{B}-C \cdot Y^{T}\right)$. Next replace $Z$ by $X_{1} \cdot Z \cdot Z_{1}^{T}$; this has the effect of changing basis in $C_{2}$. Finally, change basis in $C_{1}$ by successively replacing $\tilde{B}$ by $X_{2} \cdot \tilde{B} \cdot\left(X_{1}^{T}\right)^{-1}+C$, and then $C$ by $Y^{-1} \cdot X_{1}^{-1} \cdot C \cdot X_{2}^{T}$. Then $Z$ becomes

$$
\Delta \cdot \tilde{B}-C \cdot \Delta=\left(d_{i} b_{i j}-d_{j} c_{i j}\right) .
$$

The lemma follows immediately.

Proof of Proposition 7.8 We have $H C_{2}^{\operatorname{lin}}(K)=\operatorname{ker} M_{2}$ and $H C_{1}^{\operatorname{lin}}(K)=$ $\left(\operatorname{ker} M_{1}\right) /\left(\operatorname{im} M_{2}\right)$. Write $M_{2}$ as in Lemma [7.9] Since $d_{1}, \ldots, d_{n-1}>0, M_{2}$ has only one 0 diagonal entry, and so $\operatorname{ker} M_{2} \cong \mathbb{Z}$.

For $H C_{1}^{\text {lin }}(K)$, assume that we have chosen bases for $C_{1}, C_{2}$ so that $M_{2}$ is diagonal with diagonal entries $\delta_{1}, \ldots, \delta_{2 n^{2}}$, with $\delta_{2 n^{2}}=0$ and $\delta_{i} \neq 0$ for $i<2 n^{2}$. Let the corresponding basis for $C_{1}$ be $v_{1}, \ldots, v_{3 n^{2}-n}$. Then $v_{1}, \ldots, v_{2 n^{2}-1} \in \operatorname{ker} M_{1}$, and indeed we may assume that $\left\{v_{1}, \ldots, v_{2 n^{2}-1+m}\right\}$ forms a basis for $\operatorname{ker} M_{1}$ for some $m \geq 0$. It follows that $\left(\operatorname{ker} M_{1}\right) /\left(\operatorname{im} M_{2}\right) \cong \mathbb{Z}^{m} \oplus \oplus_{i} \mathbb{Z}_{\delta_{i}}$. From Lemma 7.9 we conclude that $\left(\operatorname{ker} M_{1}\right) /\left(\operatorname{im} M_{2}\right) \cong\left(H_{1}\left(\Sigma_{2}\right) \oplus \mathbb{Z}\right) \otimes\left(H_{1}\left(\Sigma_{2}\right) \oplus \mathbb{Z}\right) \oplus \mathbb{Z}^{m}$, as desired.

We conjecture that $m$ is always 0 in Proposition 7.8 Even if this is not the case, Proposition [7.8 still implies that we can deduce $H_{1}\left(\Sigma_{2}\right)$ from $H C_{1}^{\operatorname{lin}}(K)$; this is an easy consequence of the fundamental theorem of finitely generated abelian groups.

Corollary 7.10 The group $H_{1}\left(\Sigma_{2}\right)$, and hence the determinant $\left|\Delta_{K}(-1)\right|$, is determined by the equivalence class of the knot DGA of $K$.

We now turn our attention to $H C_{0}^{\operatorname{lin}}(K)$, and derive a result which will be used in the sequel [19].

Proposition 7.11 There are surjections

$$
H C_{0}^{\operatorname{lin}}(K) \rightarrow H C_{0}^{\mathrm{lin}, \mathrm{ab}}(K) \rightarrow \operatorname{Sym}^{2}\left(H_{1}\left(\Sigma_{2}\right)\right) .
$$


The proof of Proposition 7.11 is essentially a linearized version of the (more involved) proof of Theorem [7.1 and we provide just an outline here. The matrix $\operatorname{Bur}_{\hat{B}}$ acts on $\mathbb{Z}^{n}$, which has generators $e_{1}, \ldots, e_{n}$, and it is an easy exercise to check that $H_{1}\left(\Sigma_{2}\right)$ is generated by the differences $\left\{e_{i}-e_{j}\right\}$, modulo the relations $\operatorname{Bur}_{\hat{B}} e_{i}-e_{i}=0$ for all $i$. (Note that $\operatorname{Bur}_{\hat{B}} e_{i}-e_{i}$ is a linear combination of differences $e_{j_{1}}-e_{j_{2}}$.)

Lemma 7.12 In the ring $\mathbb{Z}\left[e_{1}, \ldots, e_{n}\right]$, if $A=\left(-2+\left(e_{i}-e_{j}\right)^{2}\right)$, then

$$
\Phi_{B}^{L}(A) \cdot A=\left(-2+\left(\operatorname{Bur}_{\hat{B}} e_{i}-e_{j}\right)^{2}+O\left(e^{4}\right)\right)
$$

and

$$
\Phi_{B}^{R}(A) \cdot A=\left(-2+\left(e_{i}-\operatorname{Bur}_{\hat{B}} e_{j}\right)^{2}+O\left(e^{4}\right)\right) .
$$

Proof Induction on the braid word $B$, as in the proof of Lemma 7.5

Proof of Proposition 7.11 The surjection from $H C_{0}^{\operatorname{lin}}(K)$ to $\operatorname{Sym}^{2}\left(H_{1}\left(\Sigma_{2}\right)\right)$ which factors through $H C_{0}^{\text {lin,ab }}(K)$, sends $a_{i j}$ to $\left(e_{i}-e_{j}\right)^{2}$. This is well-defined by Lemma 7.12 and the fact that $\operatorname{Bur}_{\hat{B}} e_{i}-e_{i}=0$ in $H_{1}\left(\Sigma_{2}\right)$ for all $i$; it is a surjection by polarization, since 2 is invertible in $\operatorname{Sym}^{2}\left(H_{1}\left(\Sigma_{2}\right)\right)$.

A more natural way to see the second surjection in Proposition 7.11 using cords, is given in Section 5.2 of [19. By all computational indications, it seems likely that this surjection is always an isomorphism, and hence that $H C_{0}^{\text {lin,ab }}(K)$ is determined by $H_{1}\left(\Sigma_{2}\right)$ for all knots $K$. However, $H C_{0}^{\operatorname{lin}}(K)$ is not determined by $H_{1}\left(\Sigma_{2}\right)$. For instance, the knots $8_{18}$ and $9_{37}$ both satisfy $H_{1}\left(\Sigma_{2}\right) \cong \mathbb{Z}_{3} \oplus \mathbb{Z}_{15}$, but $H C_{0}^{\operatorname{lin}}\left(8_{18}\right) \cong \mathbb{Z}_{3}^{3} \oplus \mathbb{Z}_{15}$, while $H C_{0}^{\operatorname{lin}}\left(9_{37}\right) \cong \mathbb{Z}_{3}^{2} \oplus \mathbb{Z}_{15}$. It is possible that $H C_{0}^{\operatorname{lin}}(K)$ is always isomorphic to either $\operatorname{Sym}^{2}\left(H_{1}\left(\Sigma_{2}\right)\right)$ or $H_{1}\left(\Sigma_{2}\right) \otimes H_{1}\left(\Sigma_{2}\right)$; this has been verified for all prime knots with eleven or fewer crossings.

\section{Computations}

The combinatorial nature of our invariants allows them to be calculated readily by computer. Mathematica source to compute the invariants is available at the author's web site. The full program requires the installation of the noncommutative algebra package NCAlgebra/NCGB [11], though there is a commutative version which does not use NCAlgebra. Using the program, we can calculate the braid homomorphism $\phi$, braid and knot DGAs, $H C_{0}$ (via Gröbner bases), augmentation numbers, and linearized knot contact homology. Many of the calculations in this section were performed with the help of the program. 


\subsection{Braid conjugacy classes}

It can be difficult to tell if two braids have equivalent braid DGAs. Currently, the most effective computational tool in this regard is the augmentation number invariant described in Section 6.3, which can be calculated by computer and is reasonably effective in distinguishing between braid conjugacy classes.

\begin{tabular}{|c||c|c|c|c|c|}
\hline$B$ & $w$ & $\operatorname{Aug}(B, 2)$ & $\operatorname{Aug}^{\mathrm{ab}}(B, 2)$ & $\mathrm{Aug}^{\mathrm{ab}}(B, 5)$ & $\mathrm{Aug}^{\mathrm{ab}}(B, 7)$ \\
\hline \hline$\sigma_{1}^{5} \sigma_{2}$ & 6 & 5 & 1 & 4 & 6 \\
\hline$\sigma_{1}^{3} \sigma_{2}^{3}$ & 6 & 9 & 5 & 12 & 18 \\
\hline$\sigma_{1}^{3} \sigma_{2}$ & 4 & 4 & 2 & 5 & 7 \\
\hline$\sigma_{1}^{5} \sigma_{2}^{-1}$ & 4 & 4 & 2 & 5 & 6 \\
\hline$\sigma_{1}^{3} \sigma_{2} \sigma_{1}^{-1} \sigma_{2}$ & 4 & 4 & 2 & 4 & 7 \\
\hline$\sigma_{1} \sigma_{2}$ & 2 & 4 & 2 & 5 & 7 \\
\hline$\sigma_{1}^{3} \sigma_{2}^{-1}$ & 2 & 4 & 2 & 4 & 7 \\
\hline$\sigma_{1}^{3} \sigma_{2}^{-1} \sigma_{1} \sigma_{2}^{-1}$ & 2 & 2 & 2 & 9 & 4 \\
\hline$\sigma_{1} \sigma_{2}^{-1}$ & 0 & 5 & 1 & 4 & 6 \\
\hline$\sigma_{1} \sigma_{2}^{-1} \sigma_{1} \sigma_{2}^{-1}$ & 0 & 9 & 5 & 12 & 18 \\
\hline$\sigma_{1}^{3} \sigma_{2}^{-3}$ & 0 & 7 & 5 & 11 & 13 \\
\hline$\sigma_{1}^{2} \sigma_{2}^{-2} \sigma_{1} \sigma_{2}^{-1}$ & 0 & 1 & 1 & 4 & 2 \\
\hline$\sigma_{1}^{-2} \sigma_{2}^{2} \sigma_{1}^{-1} \sigma_{2}$ & 0 & 1 & 1 & 4 & 2 \\
\hline
\end{tabular}

Table 1: The conjugacy classes in $B_{3}$ with nonnegative writhe which can be represented by a connected braid of word length at most 6 , identified by such a representative $B$. The writhe $w$ is given, along with various augmentation numbers.

In Table 1. we list all conjugacy classes in $B_{3}$ of nonnegative writhe which can be represented by a connected braid of word length at most 6 , along with their writhe and selected augmentation numbers. (Recall that the writhe $w$ is the homomorphism $B_{n} \rightarrow \mathbb{Z}$ for which $w\left(\sigma_{k}\right)=1$ for all $k$.) The augmentation numbers, combined with the writhe, distinguish all of the given conjugacy classes, with the exception of the mirrors $\sigma_{1}^{2} \sigma_{2}^{-2} \sigma_{1} \sigma_{2}^{-1}$ and $\sigma_{1}^{-2} \sigma_{2}^{2} \sigma_{1}^{-1} \sigma_{2}$, which by Proposition 6.8 cannot be distinguished using our invariants.

Without the use of the writhe, our invariants become less effective. For instance, it can be shown that the braid DGAs for $\sigma_{1} \sigma_{2}$ and $\sigma_{1}^{3} \sigma_{2}$ are both stable tame isomorphic to the DGA on two generators of degree 0 and two generators of degree 1 , with $\partial=0$. 


\subsection{The unknot and unlink, and other links}

The unknot is the closure of the trivial braid in $B_{1}$, whose knot DGA is generated by $b_{11}, c_{11}$ in degree 1 and $d_{11}, e_{1}$ in degree 2 , with differential given by $\partial b_{11}=\partial c_{11}=\partial d_{11}=0, \partial e_{1}=b_{11}+c_{11}$. (The $1 \times 1$ matrices $\Phi^{L}$ and $\Phi^{R}$ for the trivial braid in $B_{1}$ are simply (1).) This is stable tame isomorphic to the DGA with generators $b_{11}$ in degree 1 and $d_{11}$ in degree 2 , and with $\partial=0$. In particular, we see that $H C_{0}$ (unknot) $\cong \mathbb{Z}$.

More generally, let $L_{n}$ denote the $n$-component unlink, which is the closure of the trivial braid $B_{n}^{0}$ in $B_{n}$. The matrices $\Phi_{B_{n}^{0}}^{L}$ and $\Phi_{B_{n}^{0}}^{R}$ are both the identity, and so the knot DGA of $B_{n}^{0}$ has differential $\partial a_{i j}=\partial b_{i j}=\partial c_{i j}=\partial d_{i j}=0$, $\partial e_{i}=b_{i i}+c_{i i}$, and $H C_{0}\left(L_{n}\right) \cong \mathbb{Z}\left\langle\left\{a_{i j}\right\}\right\rangle$.

By contrast, the Hopf link, which is the closure of $\sigma_{1}^{2} \in B_{2}$, has knot DGA satisfying

$$
\partial B=\left(\begin{array}{cc}
-4+a_{12} a_{21} & 4 a_{12}-a_{12} a_{21} a_{12} \\
0 & -4+a_{21} a_{12}
\end{array}\right)
$$

and

$$
\partial C=\left(\begin{array}{cc}
-4+a_{12} a_{21} & 0 \\
4 a_{21}-a_{21} a_{12} a_{21} & -4+a_{21} a_{12}
\end{array}\right) .
$$

It follows that $H C_{0}$ (Hopf) $\cong \mathbb{Z}\left\langle a_{12}, a_{21}\right\rangle /\left\langle-4+a_{12} a_{21},-4+a_{21} a_{12}\right\rangle$, which is not isomorphic to $\mathrm{HC}_{0}\left(L_{2}\right)$.

Finally, consider the split link $3_{1} \sqcup 0_{1}$ given by the closure of $\sigma_{1}^{3} \in B_{3}$, which is the unlinked union of an unknot and a trefoil. We can compute that

$$
\begin{aligned}
H C_{0}\left(3_{1} \sqcup 0_{1}\right) \cong & \mathbb{Z}\left\langle a_{12}, a_{13}, a_{31}, a_{23}, a_{32}\right\rangle /\left\langle-2+a_{12}+a_{12}^{2},\right. \\
& \left.a_{13}-a_{23}-a_{12} a_{13}+a_{12} a_{23}, a_{31}-a_{32}-a_{31} a_{12}+a_{32} a_{12}\right\rangle,
\end{aligned}
$$

which is not isomorphic to $H C_{0}\left(L_{2}\right)$. Hence our invariants, in contrast to the Alexander polynomial, can distinguish between split links.

\subsection{Torus knots $T(2, n)$}

In this section, we generalize our computation in Section 4.1 of $H C_{0}$ for the trefoil, by computing $H C_{0}$ for all torus knots of the form $T(2, n)$.

First we introduce two sequences related to the polynomials $q_{m}(x)$ discussed in Section [7.1. In $\mathcal{A}_{2}$, define $\left\{q_{m}^{(1)}\right\},\left\{q_{m}^{(2)}\right\}$ by $q_{0}^{(1)}=q_{0}^{(2)}=-2, q_{1}^{(1)}=a_{12}$, $q_{1}^{(2)}=a_{21}$, and $q_{m+1}^{(1)}=-a_{12} q_{m}^{(2)}-q_{m-1}^{(1)}, q_{m+1}^{(2)}=-a_{21} q_{m}^{(1)}-q_{m-1}^{(2)}$; by definition, we have $\left.q_{m}^{(1)}\right|_{a_{12}=a_{21}=-x}=\left.q_{m}^{(2)}\right|_{a_{12}=a_{21}=-x}=q_{m}(x)$. 
Lemma 8.1 For $k \geq 1$, we have

$$
\Phi_{\sigma_{1}^{2 k-1}}^{L}(A) \cdot A=\left(\begin{array}{cc}
q_{2 k-1}^{(2)} & q_{2 k}^{(2)} \\
q_{2 k-2}^{(1)} & q_{2 k-1}^{(1)}
\end{array}\right) \quad \text { and } \quad A \cdot \Phi_{\sigma_{1}^{2 k-1}}^{R}(A)=\left(\begin{array}{cc}
q_{2 k-1}^{(1)} & q_{2 k-2}^{(1)} \\
q_{2 k}^{(2)} & q_{2 k-1}^{(2)}
\end{array}\right)
$$

Proof We prove the first identity; the second follows similarly or by conjugation symmetry. For $k=1$, the identity can be verified directly. For $k \geq 2$, by Proposition 4.4 we have

$$
\Phi_{\sigma_{1}^{2 k-1}}^{L}(A) \cdot A=\Phi_{\sigma_{1}^{2}}^{L}\left(\phi_{\sigma_{1}^{2 k-3}}(A)\right) \cdot \Phi_{\sigma_{1}^{2 k-3}}^{L}(A) \cdot A .
$$

Now $\phi_{\sigma_{1}^{2}}(A)=A$, and so $\phi_{\sigma_{1}^{2 m-1}}(A)=\phi_{\sigma_{1}}(A)=\left(\begin{array}{cc}-2 & a_{21} \\ a_{12} & -2\end{array}\right)$; then we compute that $\Phi_{\sigma_{1}^{2}}^{L}\left(\phi_{\sigma_{1}^{2 m-1}}(A)\right)=\left(\begin{array}{cc}-1+a_{21} a_{12} & a_{21} \\ -a_{12} & -1\end{array}\right)$. The desired identity can now be checked by induction on $m$.

We can now compute $H C_{0}$ for the $(2,2 k-1)$ torus knot.

Proposition 8.2 For $k \geq 1, H C_{0}(T(2,2 k-1)) \cong \mathbb{Z}[x] /\left(p_{k}(x)\right)$, where $p_{k}$ is the polynomial defined in Section [7.1.

Proof By Lemma 8.1] we have $\left(\left(1-\Phi_{\sigma_{1}^{2 k-1}}^{L}(A)\right) \cdot A\right)_{12}=a_{12}-q_{2 k}^{(2)}$ and $(A \cdot(1-$ $\left.\left.\Phi_{\sigma_{1}^{2 k-1}}^{R}(A)\right)\right)_{21}=a_{21}-q_{2 k}^{(2)}$. Hence in $H C_{0}(T(2,2 k-1))$, we have $a_{12}=a_{21}$ and we can set $x:=-a_{12}=-a_{21}$. The relations in $H C_{0}(T(2,2 k-1))$ then become the entries of the matrix

$$
\left(\begin{array}{cc}
-2-q_{2 k-1}(x) & -x-q_{2 k}(x) \\
-x-q_{2 k-2}(x) & -2-q_{2 k-1}(x)
\end{array}\right) .
$$

Now define $r_{m}(x)=q_{m}(x)-q_{2 k-1-m}(x)$ for all $m$, so that $r_{m}(x)=x r_{m+1}(x)-$ $r_{m+2}(x)$. We have

$$
\begin{aligned}
H C_{0}(T(2,2 k-1)) & \cong \mathbb{Z}[x] /\left(-x-q_{2 k-2}(x),-x-q_{2 k}(x),-2-q_{2 k-1}(x)\right) \\
& =\mathbb{Z}[x] /\left(r_{0}(x), r_{1}(x)\right),
\end{aligned}
$$

where the second equality holds because $-x-q_{2 k}(x)=r_{-1}(x)=x r_{0}(x)-r_{1}(x)$. Now

$$
\operatorname{gcd}\left(r_{0}(x), r_{1}(x)\right)=\operatorname{gcd}\left(r_{1}(x), r_{2}(x)\right)=\cdots=\operatorname{gcd}\left(r_{k-1}(x), r_{k}(x)\right)=r_{k-1}(x) ;
$$

since $r_{k-1}(x)=p_{k}(x)$ by induction on $k$, the proposition follows. 
Proposition 8.2 shows that the surjection in Theorem 7.1 is actually an isomorphism for $(2, n)$ torus knots. This is established in more generality, for all two-bridge knots, in [19]. However, we will see in the following sections that it is not true for all knots.

Before moving on, we make an observation regarding the trefoil in the context of Legendrian knot theory. We have seen that $H C_{0}$ for the trefoil is $\mathbb{Z}[x] /\left(x^{2}+\right.$ $x-2)$. This has two maps to $\mathbb{Z}_{2}$ and thus the knot DGA for the trefoil has two augmentations over $\mathbb{Z}_{2}$. If we use the form for the trefoil knot DGA given at the end of Section 2.2, then one augmentation, $\epsilon_{0}$, sends $a_{12}$ and $a_{21}$ to 0 , while the other, $\epsilon_{1}$, sends them to 1 . The linearized differential for $\epsilon_{0}$ is simply given by the linear terms in the differential mod 2 , and one can calculate that this has Poincaré polynomial $\lambda^{2}+\lambda$ (ie, homology of dimension 1 in degrees 2 and $1)$. On the other hand, one can check that the augmentation $\epsilon_{1}$ yields Poincaré polynomial $4 \lambda^{2}+4 \lambda$. This is an example where different augmentations of a DGA measuring relative contact homology give different Poincaré polynomials over $\mathbb{Z}_{2} ;$ this phenomenon has also now been observed for some Legendrian knots in $\mathbb{R}^{3}$ [17.

\subsection{Torus knots $T(3, n)$}

The invariant $H C_{0}$ is not as simple for general torus knots as it is for $T(2, n)$. For instance, if we write the torus knot $T(3,4)$ as the closure of the braid $\left(\sigma_{1} \sigma_{2}\right)^{4} \in B_{3}$, we can calculate

$$
\begin{aligned}
H C_{0}(T(3,4)) \cong \mathbb{Z} & \left\langle a_{1}, a_{2}\right\rangle /\left\langle 2 a_{1}-2 a_{2}-a_{1}^{2}+a_{1} a_{2}+a_{1}^{3}-a_{1}^{2} a_{2},\right. \\
& -a_{1} a_{2}+a_{2} a_{1}+a_{1}^{2} a_{2}-a_{2} a_{1}^{2},-3 a_{1}+3 a_{2}+a_{1} a_{2} a_{1}-a_{2} a_{1} a_{2}, \\
& \left.-a_{1}+a_{2}+a_{1}^{2}-a_{2}^{2}, 2+a_{2}-a_{1} a_{2}-a_{2} a_{1}-a_{1}^{3}\right\rangle .
\end{aligned}
$$

Furthermore, if we use lexicographic order with $a_{1}<a_{2}$, then the above presentation gives a Gröbner basis for the ideal. Thus as an additive abelian group, $H C_{0}(T(3,4)) \cong \mathbb{Z}^{7}$, generated by $1, a_{1}, a_{2}, a_{1}^{2}, a_{1} a_{2}, a_{2} a_{1}, a_{1} a_{2} a_{1}$. On the other hand, the abelianization of $H C_{0}(T(3,4))$ is easily shown to be isomorphic to $\mathbb{Z}[x] /\left(x^{3}-2 x^{2}-x+2\right)$, and so $H C_{0}(T(3,4))$ is noncommutative. Note that $H C_{0}(T(3,4))$ does map surjectively to $\mathbb{Z}[x] /\left(x^{2}-x-2\right)$, as per Theorem 7.1

In general, it seems that $H C_{0}(T(m, n))$ is too complicated to be expressed in closed form. However, for $T(3, n)$, there is a relatively simple expression for $H C_{0}^{\text {ab }}$, if not for $H C_{0}$ (cf $H C_{0}(T(3,4))$ above).

It is easy to see that $H C_{0}^{\mathrm{ab}}(T(3, n))$ can be written in the form $\mathbb{Z}[x] /(p(x))$ for some polynomial $p$. The knot $T(3, n)$ is the closure of $\left(\sigma_{1} \sigma_{2}\right)^{n} \in B_{3}$, and 
$\phi_{\left(\sigma_{1} \sigma_{2}\right)^{n}}$ cyclically permutes $a_{12}, a_{23}, a_{31}$ when 3 does not divide $n$. Hence the generators $a_{12}, a_{23}, a_{13}$ of $H C_{0}^{\mathrm{ab}}(T(3, n))$ are equal by Corollary 4.9, (The same argument shows that $H C_{0}(T(3, n))$ is generated by two elements.)

Explicitly calculating $H C_{0}^{\mathrm{ab}}(T(3, n))$ is more involved; a straightforward but tedious induction, along the lines of the proof of Proposition [8.2, yields the following result.

Proposition 8.3 If $\operatorname{gcd}(3, n)=1$, then

$$
H C_{0}^{\mathrm{ab}}(T(3, n)) \cong \begin{cases}\mathbb{Z}[x] /\left(r_{k}(x)\right), & n=2 k-1 \\ \mathbb{Z}[x] /\left(s_{k}(x)\right), & n=2 k-2,\end{cases}
$$

where $r_{0}(x)=2-x, r_{1}(x)=x-2, r_{m+1}(x)=(x-1) r_{m}(x)-r_{m-1}(x)$, and $s_{1}(x)=0, s_{2}(x)=(x-2)(x+1), s_{m+1}(x)=(x-1) s_{m}(x)-s_{m-1}(x)$.

Note that $r_{k}(x)=\frac{x-2}{x-3} p_{k}(x-1)$.

\subsection{The connected sums $3_{1} \# 3_{1}$ and $3_{1} \# \overline{3_{1}}$}

When we view the connected sum $3_{1} \# 3_{1}$ as the closure of $\sigma_{1}^{3} \sigma_{2}^{3} \in B_{3}$, we can calculate that $\operatorname{Aug}^{\mathrm{ab}}\left(3_{1} \# 3_{1}, 2\right)=7$, and hence $H C_{0}\left(3_{1} \# 3_{1}\right)$ is not of the form $\mathbb{Z}[x] /(p(x))$. To garner more information, we use the standard Gröbner basis algorithm to find that

$$
\begin{aligned}
H C_{0}\left(3_{1} \# 3_{1}\right) \cong \mathbb{Z} & \left\langle a_{1}, a_{2}, a_{3}, a_{4}\right\rangle /\left\langle\left(a_{1}+2\right)\left(a_{1}-1\right),\left(a_{2}+2\right)\left(a_{2}-1\right),\right. \\
& \left.\left(a_{1}-1\right) a_{3}, a_{4}\left(a_{1}-1\right),\left(a_{2}-1\right) a_{4}, a_{3}\left(a_{2}-1\right)\right\rangle .
\end{aligned}
$$

The given presentation for the above ideal is a noncommutative Gröbner basis for the ideal with respect to any lexicographic order, and it follows that $H C_{0}\left(3_{1} \# 3_{1}\right)$ is noncommutative (for instance, $a_{1} a_{3} \neq a_{3} a_{1}$ ) and not finitely generated as a $\mathbb{Z}$-module. It is also true that $H C_{0}^{\text {ab }}$ has infinite rank in this case; in fact,

$$
\begin{aligned}
H C_{0}^{\mathrm{ab}}\left(3_{1} \# 3_{1}\right) \cong & \mathbb{Z}\left[a_{1}, a_{2}, a_{3}\right] /\left(\left(a_{1}+2\right)\left(a_{1}-1\right),\left(a_{2}+2\right)\left(a_{2}-1\right),\right. \\
& \left.\left(a_{1}-1\right)\left(a_{3}-a_{2}\right),\left(a_{2}-1\right)\left(a_{3}-a_{1}\right)\right) .
\end{aligned}
$$

As another application of this example, it turns out that precisely the same computation holds for $3_{1} \# \overline{3_{1}}$; hence it is not true that $H C_{0}$ or $H C_{0}^{\text {ab }}$ determines the isotopy class of a knot, even up to mirrors. 


\subsection{Knot contact homology and other invariants}

From the computations of the previous sections, the $H C_{0}$ invariant for knots depends on more than the determinant of the knot. It is also not determined by the Alexander polynomial; for instance, the knots $6_{1}$ and $9_{46}$ have the same Alexander polynomial, but $\operatorname{Aug}^{\mathrm{ab}}\left(6_{1}, 2\right)=2$ while $\operatorname{Aug}^{\mathrm{ab}}\left(9_{46}, 2\right)=5$.

More generally, knot contact homology seems to behave independently of many known knot invariants. In particular, $H C_{0}$ can distinguish knots which share many of the same "classical" invariants.

The knots $10_{25}$ and $\overline{10_{56}}$ (the mirror of $10_{56}$, with the conventions of [22]) are both alternating, with the same HOMFLY polynomial and signature; hence they also share the same Alexander polynomial, Jones polynomial, Khovanov invariant [13] (by a result of [15]), and Ozsváth-Szabó invariant 21]. On the other hand, we have $\operatorname{Aug}^{\mathrm{ab}}\left(10_{25}, 7\right)=1$, while $\operatorname{Aug}^{\mathrm{ab}}\left(\overline{10_{56}}, 7\right)=2$.

Similarly, the knots $11_{255}^{a}$ and $11_{257}^{a}$, alternating 11 -crossing knots from Conway's enumeration [4, have the same (two-variable) Kauffman polynomial, but $\operatorname{Aug}^{\mathrm{ab}}\left(11_{255}^{a}, 7\right)=1$ while $\operatorname{Aug}^{\mathrm{ab}}\left(11_{257}^{a}, 7\right)=2$. We conclude the following result.

Proposition 8.4 The invariant $H C_{0}$ for knots is not determined by any of the following: Alexander polynomial, Jones polynomial, HOMFLY polynomial, Kauffman polynomial, signature, Khovanov invariant, and Ozsváth-Szabó invariant.

\section{An alternate knot invariant}

As explained in Section 3 in the symplectic world, the knot DGA counts holomorphic disks with certain boundary conditions determined by the knot. This count is well defined modulo 2 , and so we obtain a DGA over $\mathbb{Z}_{2}$. To lift this DGA to $\mathbb{Z}$ coefficients, however, we need a set of coherent orientations for the relevant moduli spaces. The knot DGA we have been using presumably corresponds to a choice of coherent orientations, but there are others.

By experimenting with various choices of signs, one can derive several inequivalent choices for DGAs over $\mathbb{Z}$ which are knot invariants and agree with the original knot DGA modulo 2, but only two seem to give "nice" results for simple knots (eg, such that $H C_{0}$ for small knots is of the form $\left.\mathbb{Z}[x] /(p(x))\right)$. One is the knot DGA already described; the other we call the alternate knot DGA. 
This alternate theory lacks some of the nice properties of the knot DGA - for instance, no obvious analogue of Theorem 7.1 exists for the alternate DGA - but it seems to give a sharper knot invariant than the usual DGA.

\subsection{The alternate DGA}

We follow the definition of the knot DGA, making adjustments where necessary.

Let $\tilde{\phi}$ denote the homomorphism from $B_{n}$ to $\operatorname{Aut}\left(\mathcal{A}_{n}\right)$ defined on generators by

$$
\tilde{\phi}_{\sigma_{k}}:\left\{\begin{array}{clrl}
a_{k i} & \mapsto-a_{k+1, i}+a_{k+1, k} a_{k i} & & i \neq k, k+1 \\
a_{i k} & \mapsto-a_{i, k+1}-a_{i k} a_{k, k+1} & i \neq k, k+1 \\
a_{k+1, i} & \mapsto a_{k i} & i \neq k, k+1 \\
a_{i, k+1} & \mapsto a_{i k} & i \neq k, k+1 \\
a_{k, k+1} & \mapsto-a_{k+1, k} & \\
a_{k+1, k} & \mapsto-a_{k, k+1} & i, j \neq k, k+1 . \\
a_{i j} & \mapsto a_{i j} &
\end{array}\right.
$$

Note that $\tilde{\phi}$ is identical to $\phi$ modulo 2 , and that $\phi$ and $\tilde{\phi}$ are conjugate through the automorphism of $\mathcal{A}_{n}$ which sends $a_{i j}$ to $a_{i j}$ if $i<j$ and $-a_{i j}$ if $i>j$.

Write $A=\left(a_{i j}\right), B=\left(b_{i j}\right), C=\left(c_{i j}\right), D=\left(d_{i j}\right)$ as usual, with the crucial difference that we set $a_{i i}=0$, rather than $a_{i i}=-2$, for all $i$. Denote by $\tilde{\phi}^{\text {ext }}$ the composition map $B_{n} \hookrightarrow B_{n+1} \stackrel{\tilde{\phi}}{\rightarrow} \operatorname{Aut}\left(\mathcal{A}_{n}\right)$, and define matrices $\tilde{\Phi}_{B}^{L}(A)$, $\tilde{\Phi}_{B}^{R}(A)$ by

$$
\tilde{\phi}_{B}^{\text {ext }}\left(a_{i *}\right)=\sum_{j=1}^{n}\left(\tilde{\Phi}_{B}^{L}(A)\right)_{i j} a_{j *} \quad \text { and } \quad \tilde{\phi}_{B}^{\text {ext }}\left(a_{* j}\right)=\sum_{i=1}^{n} a_{* i}\left(\tilde{\Phi}_{B}^{R}(A)\right)_{i j} .
$$

Then the alternate knot $D G A$ of $B \in B_{n}$ is generated, as before, by $\left\{a_{i j} \mid 1 \leq\right.$ $i \neq j \leq n\}$ in degree $0,\left\{b_{i j}, c_{i j} \mid 1 \leq i, j \leq n\right\}$ in degree 1 , and $\left\{d_{i j} \mid 1 \leq i, j \leq\right.$ $n\}$ and $\left\{e_{i} \mid 1 \leq i \leq n\right\}$ in degree 2 , with

$$
\begin{aligned}
\partial A & =0 \\
\partial B & =\left(1+\tilde{\Phi}_{B}^{L}(A)\right) \cdot A \\
\partial C & =A \cdot\left(1+\tilde{\Phi}_{B}^{R}(A)\right) \\
\partial D & =B \cdot\left(1+\tilde{\Phi}_{B}^{R}(A)\right)-\left(1+\tilde{\Phi}_{B}^{L}(A)\right) \cdot C \\
\partial e_{i} & =\left(B-\tilde{\Phi}_{B}^{L}(A) \cdot C\right)_{i i} .
\end{aligned}
$$

Note the difference in signs from Definition 2.9. Also, let the alternate contact homology of a knot $K$, written $\widetilde{H C}_{*}(K)$, be the homology of the alternate 
knot DGA of any braid closing to $K$. As mentioned before, the alternate knot DGA and contact homology are identical modulo 2 to the usual knot DGA and contact homology.

Theorem 9.1 The stable tame isomorphism class of the alternate knot DGA, as well as $\widetilde{H C}_{*}$, are knot invariants.

The proof of Theorem 9.1 is entirely analogous to the proofs from Sections 4 and 5 , and is omitted here.

Many of the results from Section [6 still hold in this context. We can define conjugation on the alternate knot DGA by $\overline{a_{i j}}=-a_{j i}, \overline{b_{i j}}=-c_{j i}, \overline{c_{i j}}=-b_{j i}$, $\overline{d_{i j}}=d_{j i}$, and $\overline{e_{i}}=d_{i i}-e_{i}$; then the differential $\partial$ in the alternate knot DGA commutes with conjugation. Hence we can form the alternate abelian knot DGA similarly to Definition [6.5. but with $a_{j i}=-a_{i j}$ and $c_{i j}=-b_{j i}$. None of the alternate invariants distinguish between knot mirrors or inverses.

\subsection{Computations of $\widetilde{H C}_{0}(K)$ for small knots}

One interesting question is whether an analogue of Theorem[7.1] holds for $\widetilde{H C}_{0}$. In all computations performed by the author, there is a surjection from $\widetilde{H C}_{0}(K)$ to some ring of the form $\mathbb{Z}[x] /(p(x))$, where $p$ is monic of degree $\frac{n(K)+1}{2}$ and $p(x) \equiv p_{(n(K)+1) / 2}(x)(\bmod 2)$. Why this should be true in general is unclear.

Nevertheless, for small knots $K$, we have $\widetilde{H C}_{0}(K) \cong \mathbb{Z}[x] /(p(x))$ for some polynomial $p$ with $\operatorname{deg}(p)=\left(\left|\Delta_{K}(-1)\right|+1\right) / 2$. In contrast to the case of $H C_{0}$, however, the polynomial $p$ depends on more than just $\left|\Delta_{K}(-1)\right|$. For example, the knots $4_{1}$ and $5_{1}$ both satisfy $\left|\Delta_{K}(-1)\right|=5$ and $H C_{0}(K) \cong \mathbb{Z}[x] /\left(x^{3}-x^{2}-\right.$ $3 x+2)$, but $\widetilde{H C}_{0}\left(4_{1}\right) \cong \mathbb{Z}[x] /\left(x^{3}-x^{2}+x\right)$, while $\widetilde{H C}\left(5_{1}\right) \cong \mathbb{Z}[x] /\left(x^{3}-x^{2}-x\right)$. See Table 2 for more examples.

Proposition 9.2 If $K$ is a prime knot with seven or fewer crossings, then we can write $\widetilde{H C}_{0}(K) \cong \mathbb{Z}[x] /(p(x))$ with $\operatorname{deg}(p)=\left(\left|\Delta_{K}(-1)\right|+1\right) / 2$. Furthermore, $p$ can be expressed as $p=\tilde{p}_{\left(\left|\Delta_{K}(-1)\right|+1\right) / 2}$ for some sequence $\left\{\tilde{p}_{m}\right\}$ with $\tilde{p}_{0}(x)=\tilde{p}_{1}(x)=x$ and $\tilde{p}_{m+1}(x)=x \tilde{p}_{m}(x) \pm \tilde{p}_{m-1}(x)$ for each $m$; in particular, $p \equiv p_{\left(\left|\Delta_{K}(-1)\right|+1\right) / 2}(\bmod 2)$, with $\left\{p_{m}\right\}$ as in Section 7.1.

Proposition 9.2 can be verified by direct computation using the author's program in Mathematica; see Table 2 .

As with $H C_{0}$, it is easy to compute $\widetilde{H C}_{0}$ explicitly for any torus knot $T(2, n)$. 


\begin{tabular}{|l||l|r|}
\hline$K$ & \multicolumn{1}{|c|}{$p(x)$ with $\widetilde{H C}_{0}(K) \cong \mathbb{Z}[x] /(p(x))$} & $D(p)$ \\
\hline \hline $0_{1}$ & $x$ & 1 \\
\hline $3_{1}$ & $x^{2}-x$ & 1 \\
\hline $4_{1}$ & $x^{3}-x^{2}+x$ & -3 \\
\hline $5_{1}$ & $x^{3}-x^{2}-x$ & 5 \\
\hline $5_{2}$ & $x^{4}-x^{3}+x$ & -23 \\
\hline $6_{1}$ & $x^{5}-x^{4}+x^{3}+x$ & 257 \\
\hline $6_{2}$ & $x^{6}-x^{5}-2 x^{4}+x^{3}+x^{2}+x$ & 1777 \\
\hline $6_{3}$ & $x^{7}-x^{6}-x^{5}+2 x^{4}-x^{2}+x$ & -10571 \\
\hline $7_{1}$ & $x^{4}-x^{3}-2 x^{2}+x$ & 49 \\
\hline $7_{2}$ & $x^{6}-x^{5}+x^{3}+x^{2}-x$ & 4409 \\
\hline $7_{3}$ & $x^{7}-x^{6}-3 x^{5}+2 x^{4}+2 x^{3}+x^{2}-x$ & 78301 \\
\hline $7_{4}$ & $x^{8}-x^{7}+4 x^{6}-3 x^{5}+4 x^{4}-2 x^{3}+x$ & -1166607 \\
\hline $7_{5}$ & $x^{9}-x^{8}-x^{7}+2 x^{6}+x^{5}-2 x^{4}+2 x^{2}-x$ & -4690927 \\
\hline $7_{6}$ & $x^{10}-x^{9}+2 x^{8}-x^{7}+3 x^{6}-x^{5}+2 x^{4}+x^{2}+x$ & 90320393 \\
\hline $7_{7}$ & $x^{11}-x^{10}+3 x^{9}-2 x^{8}+4 x^{7}-3 x^{6}+3 x^{5}-2 x^{4}+x^{3}-x^{2}+x$ & -932501627 \\
\hline
\end{tabular}

Table 2: The invariant $\widetilde{H C}_{0}(K)$ for prime knots with seven or fewer crossings, and the discriminant $D(p)$. Knot notation is as in 22].

Proposition 9.3 For $k \geq 1, H C_{0}(T(2,2 k-1)) \cong \mathbb{Z}[x] /\left(\tilde{p}_{k}(x)\right)$, where

$$
\tilde{p}_{k}(x)=\sum_{j=0}^{k-1}(-1)^{\lceil j / 2\rceil}\left(\begin{array}{c}
k-1-\lceil j / 2\rceil \\
\lfloor j / 2\rfloor
\end{array}\right) x^{k-j} .
$$

Note that the sequence $\left\{\tilde{p}_{k}\right\}$ in Proposition 9.3 satisfies $\tilde{p}_{0}(x)=\tilde{p}_{1}(x)=x$ and $\tilde{p}_{m+1}(x)=x \tilde{p}_{m}(x)-\tilde{p}_{m-1}(x)$, and that $\tilde{p}_{m}(x)=x p_{m}(x) /(x+2)$ for all $m$.

In general, when $\widetilde{H C}_{0}(K)$ is of the form $\mathbb{Z}[x] /(p(x))$ with $p$ monic, the polynomial $p(x)$ is not unique; however, we can deduce two useful invariants of the isomorphism class of $\widetilde{H C}_{0}(K)$ from $p(x)$. The first is the degree of $p$, which is simply the vector space dimension of $\widetilde{H C}_{0}(K ; \mathbb{Q})$. The second is the discriminant $D(p)$, the well-known integer invariant which can be defined as $\prod_{i<j}\left(r_{i}-r_{j}\right)^{2}$, where $\left\{r_{i}\right\}$ is the set of roots of $p$.

Table 2 gives $\widetilde{H C}_{0}(K)$ for all prime knots $K$ with seven or fewer crossings, while 


\begin{tabular}{|l||l|l|}
\hline$K$ & \multicolumn{1}{|c|}{$p(x)$ with $H C_{0}(K) \cong \mathbb{Z}[x] /(p(x))$} & $p(x)$ with $\widetilde{H C}_{0}(K) \cong \mathbb{Z}[x] /(p(x))$ \\
\hline \hline $0_{1}$ & $x-2$ & $x$ \\
\hline $3_{1}$ & $x^{2}-x-2$ & $x^{2}-x$ \\
\hline $4_{1}$ & $x^{3}-x^{2}-3 x+2$ & $x^{3}-x^{2}+x$ \\
\hline $5_{1}$ & $x^{3}-x^{2}-3 x+2$ & $x^{3}-x^{2}-x$ \\
\hline $5_{2}$ & $x^{4}-x^{3}-4 x^{2}+3 x+2$ & $x^{4}-x^{3}+x$ \\
\hline $6_{1}$ & $x^{5}-x^{4}-5 x^{3}+4 x^{2}+5 x-2$ & $x^{5}-x^{4}+x^{3}+x$ \\
\hline $6_{2}$ & $x^{6}-x^{5}-6 x^{4}+5 x^{3}+9 x^{2}-5 x-2$ & $x^{6}-x^{5}-2 x^{4}+x^{3}+x^{2}+x$ \\
\hline $6_{3}$ & $x^{7}-x^{6}-7 x^{5}+6 x^{4}+14 x^{3}-9 x^{2}-7 x+2$ & $x^{7}-x^{6}-x^{5}+2 x^{4}-x^{2}+x$ \\
\hline $7_{1}$ & $x^{4}-x^{3}-4 x^{2}+3 x+2$ & $x^{4}-x^{3}-2 x^{2}+x$ \\
\hline $7_{2}$ & $x^{6}-x^{5}-6 x^{4}+5 x^{3}+9 x^{2}-5 x-2$ & $x^{6}-x^{5}+x^{3}+x^{2}-x$ \\
\hline $7_{3}$ & $x^{7}-x^{6}-7 x^{5}+6 x^{4}+14 x^{3}-9 x^{2}-7 x+2$ & $x^{7}-x^{6}-3 x^{5}+2 x^{4}+2 x^{3}+x^{2}-x$ \\
\hline
\end{tabular}

Table 3: Comparison between $H C_{0}$ and $\widetilde{H C}_{0}$ for some small knots.

Table 3 contrasts the invariants $H C_{0}$ and $\widetilde{H C}_{0}$. Note that the discriminant on $\widetilde{H C}_{0}$ allows us to distinguish between knots which have the same $H C_{0}$; in other words, lifting $\widehat{H C}_{0}\left(K ; \mathbb{Z}_{2}\right)=H C_{0}\left(K ; \mathbb{Z}_{2}\right)$ to $\widetilde{H C}_{0}(K)$ does give new information. This would seem to provide an instance where considering orientations in contact homology yields results not given by the unoriented theory.

\section{References}

[1] J Birman, Braids, Links, and Mapping Class Groups, Ann. of Math. Studies 82, Princeton University Press, Princeton (1974) MathReview

[2] G Burde, H Zieschang, Knots, 2nd edition, de Gruyter Studies in Mathematics 5, Walter de Gruyter, Berlin (2003) MathReview

[3] Y Chekanov, Differential algebra of Legendrian links, Invent. Math. 150 (2002) 441-483 MathReview

[4] J H Conway, An enumeration of knots and links, and some of their algebraic properties, from "Computational Problems in Abstract Algebra (Oxford, 1967)", Pergamon, Oxford (1970) 329-358 MathReview

[5] T Ekholm, J Etnyre, M Sullivan, Legendrian submanifolds in $R^{2 n+1}$ and contact homology, arXiv:math.SG/0210124 
[6] Y Eliashberg, Invariants in contact topology, from: "Proceedings of the ICM (Berlin, 1998)", Doc. Math. Extra Vol. II (1998) 327-338 MathReview

[7] Y Eliashberg, A Givental, H Hofer, Introduction to Symplectic Field Theory, GAFA 2000 (Tel Aviv, 1999), Geom. Funct. Anal. Special Volume, Part II (2000) 560-673 MathReview

[8] J Epstein, D Fuchs, M Meyer, Chekanov-Eliashberg invariants and transverse approximations of Legendrian knots, Pacific J. Math. 201 (2001) 89-106 MathReview

[9] J Etnyre, L Ng, J Sabloff, Invariants of Legendrian knots and coherent orientations, J. Symplectic Geom. 1 (2002) 321-367 MathReview

[10] K Fukaya, Y-G Oh, Zero-loop open strings in the cotangent bundle and Morse homotopy, Asian J. Math. 1 (1997) 96-180 MathReview

[11] J Helton, R Miller, M Stankus, NCAlgebra: a Mathematica package for doing non commuting algebra, http://www.math.ucsd.edu/ ncalg

[12] S Humphries, An approach to automorphisms of free groups and braids via transvections, Math. Z. 209 (1992) 131-152 MathReview

[13] M Khovanov, A categorification of the Jones polynomial, Duke Math. J. 101 (2000) 359-426 MathReview

[14] D Krammer, The braid group $B_{4}$ is linear, Invent. Math. 142 (2000) 451-486 MathReview

[15] E S Lee, On Khovanov invariant for alternating links, Adv. Math. to appear, arXiv:math.GT/0210213

[16] W Magnus, Rings of Fricke characters and automorphism groups of free groups, Math. Z. 170 (1980) 91-103 MathReview

[17] P Melvin, S Shrestha, The nonuniqueness of Chekanov polynomials of Legendrian knots, arXiv:math.GT/0411206

[18] L Ng, Computable Legendrian invariants, Topology 42 (2003) 55-82 MathReview

[19] $\mathbf{L} \mathbf{~ N g}$, Knot and braid invariants from contact homology II, preprint, arXiv:math.GT/0303343

[20] H Ooguri, C Vafa, Knot invariants and topological strings, Nuclear Phys. B 577 (2000) 419-438 MathReview

[21] P Ozsváth, Z Szabó, Holomorphic disks and knot invariants, Adv. Math. 186 (2004) 58-116 MathReview

[22] D Rolfsen, Knots and Links, Publish or Perish, Berkeley (1976) MathReview

[23] J Sabloff, Invariants of Legendrian knots in circle bundles, Commun. Contemp. Math. 5 (2003) 569-627 MathReview 